\title{
Analytic Pricing of Contingent Claims Under the Real-World Measure
}

\author{
Shane M. Miller ${ }^{1}$ and Eckhard Platen ${ }^{2}$
}

February 12, 2008

\begin{abstract}
This article derives a series of analytic formulae for various contingent claims under the real-world probability measure using the stylised minimal market model (SMMM). This model provides realistic dynamics for the growth optimal portfolio (GOP) as a well-diversified equity index. It captures both leptokurtic returns with correct tail properties and the leverage effect. Under the SMMM, the discounted GOP takes the form of a timetransformed squared Bessel process of dimension four. From this property, one finds that the SMMM possesses a special and interesting relationship to non-central chi-square random variables with zero degrees of freedom. The analytic formulae derived under the SMMM include options on the GOP, options on exchange prices and options on zero-coupon bonds. For options on zero-coupon bonds, analytic prices facilitate efficient calculation of interest rate caps and floors.
\end{abstract}

1991 Mathematics Subject Classification: primary 90A12; secondary 60G30, 62P20. JEL Classification: G10, G13

Key words and phrases: benchmark approach, real-world pricing, growth optimal portfolio, minimal market model, zero-coupon bonds, exchange prices, interest rate caps and floors.

\footnotetext{
${ }^{1}$ Citigroup Global Markets Australia Pty Ltd, 2 Park Street, Sydney NSW 2000, Australia.

${ }^{2}$ University of Technology Sydney, School of Finance \& Economics and Department of Mathematical Sciences, PO Box 123, Broadway NSW 2007, Australia.
} 


\section{Introduction}

In this paper we consider a stylised version of the minimal market model (SMMM) originally proposed in Platen (2001) for modelling of the growth optimal portfolio (GOP). The GOP achieves the maximum possible growth rate of any portfolio over the long-term and can be shown to represent the best performing portfolio in several different ways. The SMMM provides realistic dynamics for the GOP as a well-diversified total return equity index, since it captures both leptokurtic returns with correct tail properties and the well-known leverage effect. As detailed in Platen (2001), the SMMM arises from an analysis of optimal market dynamics for the GOP. If it is assumed that the drift of the discounted GOP evolves smoothly over time in an exponential form, the resulting dynamics will be that of the SMMM. Under this model, the discounted GOP takes the form of a time-transformed squared Bessel process of dimension four. From this property, we find that the SMMM possesses a special and interesting relationship to non-central chi-square random variables with zero degrees of freedom. This distribution first appears in the derivation of the real-world price of a zero-coupon bond under the SMMM. It is also relevant in all subsequent derivations in this paper, including options on the GOP, options on exchange prices and options on zero-coupon bonds. Previous analysis of options on the GOP in Heath \& Platen (2002) used numerical integration of the pricing function. The analytic results derived below allow the efficient calculation of SMMM option prices and corresponding implied volatility surfaces. In the case of options on exchange prices, we derive results in terms of doubly non-central beta random variables with degrees of freedom that are either zero or four, revealing the origin of the distribution as a ratio of non-central random variates. These results are related to the work in Hulley, Miller \& Platen (2005), where analytic prices were derived for options on primary security accounts for an SMMM that includes jumps. For options on zero-coupon bonds, analytic prices facilitate efficient calculation of interest rate caps and floors under the SMMM. The paper is structured as follows: Section 2 details the key quantities of a continuous financial market under the benchmark approach of Heath \& Platen (2006); Section 3 discusses the interest rate term structure via zero-cpoupon bonds and forward rates given the SMMM; while Section 4 concerns options on the GOP under the SMMM; Section 5 provides for options on exchange prices under the SMMM; and Section 6 details on pricing interest rate caps and floors under the SMMM via options on zero-coupon bonds.

\section{Continuous Financial Market}

We consider a continuous multi-asset market with $d+1$ primary assets. Uncertainty in this market is modelled by $d$ independent standard Wiener processes $W^{k}=\left\{W_{t}^{k}, t \in[0, T]\right\}, k \in\{1,2, \ldots, d\}$. These are defined on a filtered probability space $\left(\Omega, \mathcal{A}_{T}, \underline{\mathcal{A}}, P\right)$ with finite time horizon $T \in(0, \infty)$ and filtration 
$\underline{\mathcal{A}}=\left(\mathcal{A}_{t}\right)_{t \in[0, T]}$ fulfilling the usual conditions, as given in Karatzas \& Shreve (1998). $P$ is the real-world probability measure. In the following we summarise the benchmark approach as developed in Platen \& Heath (2006).

We start by describing the main elements of a financial market, with particular focus on the key financial quantities discussed under the benchmark approach of Platen (2002). These include savings accounts, the GOP, exchange prices, primary security accounts and the discounted GOP.

We assume that the $i$ th savings account $B_{t}^{i}$, denominated in the $i$ th currency and associated with the $i$ th short rate $r_{t}^{i}$, satisfies the equation

$$
B_{t}^{i}=B_{0}^{i} \exp \left\{\int_{0}^{t} r_{s}^{i} d s\right\}
$$

for $t \in[0, T]$ and $i \in\{0,1, \ldots, d\}$, where we set $B_{0}^{i}=1$ without loss of generality.

The growth optimal portfolio (GOP) of our continuous market achieves the maximum possible expected growth rate over any time horizon. As such, the GOP can be shown to be the best performing pathwise portfolio in the long run. It has been studied previously in Kelly (1956), Long (1990), Karatzas \& Shreve (1998), Platen (2002) and many other researchers. We define the GOP process $S^{i, \delta_{*}}=\left\{S_{t}^{i, \delta_{*}}, t \in[0, T]\right\}$ in the denomination of the $i$ th currency by the stochastic differential equation (SDE)

$$
d S_{t}^{i, \delta_{*}}=S_{t}^{i, \delta_{*}}\left[r_{t}^{i}+\left|\theta_{t}^{i}\right|^{2}\right] d t+S_{t}^{i, \delta_{*}}\left|\theta_{t}^{i}\right| d W_{t}^{i}
$$

for $t \in[0, T]$ and $i \in\{0,1, \ldots, d\}$. Here $\left|\theta^{i}\right|=\left\{\left|\theta_{t}^{i}\right|, t \in[0, T]\right\}$ indicates the total market price of risk in the $i$ th currency denomination. Also note from (2.2) that $\left|\theta_{t}^{i}\right|$ can be interpreted as the volatility of the GOP. The process $W^{i}=\left\{W_{t}^{i}, t \in\right.$ $[0, T]\}$ is a Wiener process, usually correlated with the Wiener processes driving the GOP in other currency denominations.

Platen (2005b) shows that appropriately defined well-diversified portfolios approximate the GOP and exhibit similar behaviour under a mild regularity condition. Thus commonly used stock market indices can be used to approximate the GOP, including but not limited to the MSCI Growth World Stock Index (MSCI). The implications of this Diversification Theorem in Platen (2005b) are that market observable data obtained from well-diversified stock market indices can be used in conjunction with the benchmark approach for practical applications.

The GOP is immediately useful as a means to define an exchange price $X_{t}^{i, j}$, as the amount one pays in units of the $i$ th primary asset at time $t$ to obtain one unit of the $j$ th primary asset, hence

$$
X_{t}^{i, j}=\frac{S_{t}^{i, \delta_{*}}}{S_{t}^{j, \delta_{*}}}
$$


for $t \in[0, T]$ and $i, j \in\{0,1, \ldots, d\}$. Thus exchange prices can be defined analogously to an exchange rate between different currencies. Next we introduce the primary security account process $S^{i, j}=\left\{S_{t}^{i, j}, t \in[0, T]\right\}$ for $i, j \in\{0,1, \ldots, d\}$ to model the $j$ th primary asset $j \in\{0,1, \ldots, d\}$ when measured in units of the $i$ th currency. Each primary security account represents the accumulation of all income, carrying costs plus capital gains or losses achieved whilst holding the underlying primary asset. The exchange price thus provides a link between primary security accounts and savings accounts, written as

$$
S_{t}^{i, j}=X_{t}^{i, j} B_{t}^{j}=\frac{S_{t}^{i, \delta_{*}}}{S_{t}^{j, \delta_{*}}} B_{t}^{j}
$$

for $t \in[0, T]$ and $i, j \in\{0,1, \ldots, d\}$.

Next note from (2.2) that the GOP dynamics in the $i$ th currency denomination are characterized by the short rate $r_{t}^{i}$ and the total market price of risk $\left|\theta_{t}^{i}\right|$ for $t \in[0, T]$ and $i \in\{0,1, \ldots, d\}$. We can separate these two effects by considering the discounted $G O P$ process $\bar{S}^{i, \delta_{*}}=\left\{S_{t}^{i, \delta_{*}}, t \in[0, T]\right\}$, given by

$$
\bar{S}_{t}^{i, \delta_{*}}=\frac{S_{t}^{i, \delta_{*}}}{B_{t}^{i}}
$$

satisfying the SDE

$$
d \bar{S}_{t}^{i, \delta_{*}}=\bar{S}_{t}^{i, \delta_{*}}\left|\theta_{t}^{i}\right|^{2} d t+\bar{S}_{t}^{i, \delta_{*}}\left|\theta_{t}^{i}\right| d W_{t}^{i}
$$

for $t \in[0, T]$ and $i \in\{0,1, \ldots, d\}$. Thus discounting in each currency denomination by its savings account, provides a natural way to separate the corresponding short rate and the market price of risk components of the GOP in each respective denomination.

Under the benchmark approach, real-world pricing as outlined in Platen \& Heath (2006), involves the selection of the GOP as the numeraire portfolio. This leads to the pricing of securities using conditional expectations with respect to the realworld probability measure $P$. Since all positive portfolios expressed in units of the GOP are supermartingales, Platen (2002) showed that the resulting price system does not permit arbitrage. In words, the definition of no-arbitrage used under the benchmark approach is that "strictly positive profits cannot be generated under limited liability with strictly positive probability from zero initial wealth". This definition of no-arbitrage is different to the no-free-lunch-with-vanishingrisk (NFLVR) condition of Delbaen \& Schachermayer (1994). Importantly, some realistic models exist that are excluded by the NFLVR condition. In particular, the SMMM does not satisfy this condition. The NFLVR condition seems to be too restrictive since the existence of an equivalent probability measure is not necessary to capture the economic spirit of no-arbitrage. Loewenstein \& Willard (2000) argue the same point by showing that an absence of arbitrage opportunities is 
equivalent to the existence of an optimum for a representative hypothetical agent that prefers more to less, and takes prices as given. This may also be expressed as the existence of a competitive equilibrium resulting in a price system referred to as 'viable' by Harrison \& Kreps (1979).

Now we define a contingent claim $H_{\bar{T}}^{i}$ that matures at the stopping time $\bar{T} \in[0, T]$ for each $i \in\{0,1, \ldots, d\}$ as an $\mathcal{A}_{\bar{T}}$-measureable non-negative payoff that possesses a finite expectation when benchmarked by the GOP. As outlined in Platen (2002), the value $U_{t}^{i, H_{\bar{T}}^{i}}$ in the $i$ th denomination can be obtained at time $t$ by the realworld pricing formula

$$
U_{t}^{i, H_{\bar{T}}^{i}}=E\left[\frac{S_{t}^{i, \delta_{*}}}{S_{\bar{T}}^{i, \delta_{*}}} H_{\bar{T}}^{i} \mid \mathcal{A}_{t}\right]
$$

for $t \in[0, \bar{T}]$ and $i \in\{0,1, \ldots, d\}$.

Whilst it is possible under the benchmark approach that other self-financing price processes may replicate the contingent claim $H_{\bar{T}}^{i}$, the real-world price process is the minimal replicating price process. This follows since a martingale is the minimal replicating non-negative supermartingale.

From Karatzas \& Shreve (1998) it follows that in a complete continuous market, the candidate Radon-Nikodym derivative process $\Lambda^{i, \theta}=\left\{\Lambda_{t}^{i, \theta}, t \in[0, T]\right\}$ in the $i$ th currency denomination for the putative risk-neutral measure $P_{i, \theta}$ equals

$$
\Lambda_{t}^{i, \theta}=\left.\frac{d P_{i, \theta}}{d P}\right|_{\mathcal{A}_{t}}=\frac{B_{t}^{i}}{S_{t}^{i, \delta_{*}}} \frac{S_{0}^{i, \delta_{*}}}{B_{0}^{i}}=\frac{\bar{S}_{0}^{i, \delta_{*}}}{\bar{S}_{t}^{i, \delta_{*}}}
$$

up to a constant normalisation factor, the inverse of the discounted GOP with initial value $\Lambda_{0}^{i, \theta}=1$. One can obtain the SDE for the candidate Radon-Nikodym derivative by using (2.6) and (2.8) with the Itô formula as

$$
d \Lambda_{t}^{i, \theta}=-\Lambda_{t}^{i, \theta}\left|\theta_{t}^{i}\right| d \hat{W}_{t}^{i}
$$

for $t \in[0, T]$ and $i \in\{0,1, \ldots, d\}$. Obviously, $\Lambda^{i, \theta}$ is an $(\underline{\mathcal{A}}, P)$-local martingale. Whether or not the $\operatorname{SDE}(2.9)$ describes a martingale will depend upon the nature of the volatility $\left|\theta_{t}^{i}\right|$ of the GOP. For models where the candidate risk-neutral measure $P_{i, \theta}$ and the real-world measure $P$ are equivalent, as defined in Karatzas \& Shreve (1998), the Radon-Nikodym derivative process $\Lambda^{i, \theta}$ is an $(\underline{\mathcal{A}}, P)$-martingale. Here the real-world pricing formula (2.7) simplifies via Bayes' formula and Girsanov's Theorem to the standard risk-neutral pricing formula of

$$
U_{t}^{i, H_{\bar{T}}^{i}}=E_{i, \theta}\left[\frac{B_{t}^{i}}{B_{\bar{T}}^{i}} H_{\bar{T}}^{i} \mid \mathcal{A}_{t}\right]
$$

for $t \in[0, \bar{T}]$ and $i \in\{0,1, \ldots, d\}$. Here $E_{i, \theta}$ denotes conditional expectation 
with respect to the risk-neutral probability measure $P_{i, \theta}$. However, it should be noted that for the SMMM discussed in this paper, the assumptions underlying the risk-neutral pricing formula are not satisfied, and thus (2.10) cannot be used. In contrast, the real-world pricing formula (2.7) remains applicable even when the candidate Radon-Nikodym derivative is a strict $(\underline{\mathcal{A}}, P)$-local martingale, as is the case for the SMMM.

\section{Stylised MMM Dynamics}

The stylised version of the minimal market model (SMMM) was first discussed in Platen (2001), subsequently developed in a sequence of papers for equity indices and interest rate markets summarised in Heath \& Platen (2006).

We begin by introducing the discounted GOP drift as $\bar{\alpha}^{i}=\left\{\bar{\alpha}_{t}^{i}, t \in[0, T]\right\}$ for the $i$ th denomination $i \in\{0,1, \ldots, d\}$. It then follows by (2.6) that the discounted GOP drift takes the form

$$
\bar{\alpha}_{t}^{i}=\bar{S}_{t}^{i, \delta_{*}}\left|\theta_{t}^{i}\right|^{2}
$$

for $t \in[0, T]$ and $i \in\{0,1, \ldots, d\}$. Therefore by (2.6) and (3.1) one obtains, without loss of generality, an alternative representation for the SDE of the discounted GOP as

$$
d \bar{S}_{t}^{i, \delta_{*}}=\bar{\alpha}_{t}^{i} d t+\sqrt{\bar{\alpha}_{t}^{i} \bar{S}_{t}^{i, \delta_{*}}} d W_{t}^{i}
$$

for $t \in[0, T]$ and $i \in\{0,1, \ldots, d\}$.

Empirical long term data on global total return stock indices, for instance those studied by Dimson, Marsh \& Staunton (2002), motivates us to model $\bar{\alpha}_{t}^{i}$ as a smooth, deterministic function that increases over time in an exponential manner, via the following assumption.

Assumption 3.1 The discounted GOP drift $\bar{\alpha}_{t}^{i}$ is assumed to be a differentiable function of the form

$$
\bar{\alpha}_{t}^{i}=\bar{\alpha}_{0}^{i} \exp \left\{\eta^{i} t\right\}
$$

for $t \in[0, T]$ and $i \in\{0,1, \ldots, d\}$.

Here $\eta^{i}$ is the growth rate of the discounted GOP at time $t$, which is assumed to be deterministic. Alternatively, it can be interpreted as the growth rate of the GOP achieved in excess of the short-term interest rate.

Under Assumption 3.1 the discounted GOP process is a time-transformed squared Bessel (BESQ) process of dimension four, as shown in Revuz \& Yor (1999). The underlying time change $\varphi^{i}=\left\{\varphi_{t}^{i}, t \in\left[\varphi_{0}^{i}, \varphi_{T}^{i}\right]\right\}$ is defined as

$$
\varphi_{t}^{i}=\varphi_{0}^{i}+\left\langle\bar{M}^{i}\right\rangle_{t}=\varphi_{0}^{i}+\frac{1}{4} \int_{0}^{t} \bar{\alpha}_{s}^{i} d s=\varphi_{0}^{i}+\frac{\bar{\alpha}_{0}^{i}}{4 \eta_{i}}\left(\exp \left\{\eta_{i} t\right\}-1\right)
$$


or equivalently

$$
d \varphi_{t}^{i}=\frac{1}{4} \bar{\alpha}_{t}^{i} d t
$$

for $t \in[0, T]$ and $i \in\{0,1, \ldots, d\}$. Therefore the discounted GOP process $X^{i}=$ $\left\{X_{\varphi_{t}^{i}}^{i}, \varphi_{t}^{i} \in\left[\varphi_{0}^{i}, \varphi_{T}^{i}\right]\right\}$, in the transformed time (3.4), is found by setting

$$
X_{\varphi_{t}^{i}}^{i}=\bar{S}_{t}^{i, \delta_{*}}
$$

for $t \in[0, T]$ and $i \in\{0,1, \ldots, d\}$ with the initial condition $X_{\varphi_{0}^{i}}^{i}=\bar{S}_{0}^{i, \delta_{*}}$.

From Revuz \& Yor (1999) we know that a BESQ process of dimension four will never reach zero under the real-world probability measure $P$. In contrast, under the candidate risk-neutral measure $P_{i, \theta}$ it will be absorbed at zero with strictly positive probability, since under this measure it is a time-transformed squared Bessel process of dimension zero. This indicates that the candidate riskneutral measure $P_{i, \theta}$ is not equivalent to the real-world measure $P$. In addition, the candidate Radon-Nikodym derivative $\Lambda^{i, \theta}$ is a strict $(\mathcal{A}, P)$-supermartingale. Therefore, the basic assumptions of classical risk-neutral pricing, provided in say Karatzas \& Shreve (1998), are not satisfied.

Given the parameterisation for the discounted GOP drift defined in (3.3), it is useful to introduce an analogous GOP drift term of the form

$$
\alpha_{t}^{i}=\bar{\alpha}_{t}^{i} B_{t}^{i}
$$

for $t \in[0, T]$ and $i \in\{0,1, \ldots, d\}$, using (2.1). Thus by (2.1), (3.2) and (3.7) we can provide the SMMM representation for the SDE of the GOP as

$$
d S_{t}^{i, \delta_{*}}=\left(r_{t}^{i} S_{t}^{i, \delta_{*}}+\alpha_{t}^{i}\right) d t+\sqrt{\alpha_{t}^{i} S_{t}^{i, \delta_{*}}} d W_{t}^{i}
$$

for $t \in[0, T]$ and $i \in\{0,1, \ldots, d\}$. Therefore under the SMMM, the GOP is modelled as a generalised square root process.

We now re-write the volatility of the GOP using (2.2), (2.5), (3.2) and (3.8) to find

$$
\left|\theta_{t}^{i}\right|=\sqrt{\frac{\bar{\alpha}_{t}^{i}}{\bar{S}_{t}^{i, \delta_{*}}}}=\sqrt{\frac{\alpha_{t}^{i}}{S_{t}^{i, \delta_{*}}}}
$$

for $t \in[0, T]$ and $i \in\{0,1, \ldots, d\}$. This expression for GOP volatility can be interpreted as a local volatility function characterisation of the SMMM. The dynamics for the volatility of the GOP under the SMMM given in (3.9) are stationary, and thus always remain within a realistic range. To illustrate this fact, we calculated a historical time series for the volatility of the GOP using the World Stock Accumulation Index (WSAI) provided by Global Financial Data to 
approximate the GOP, as plotted in Figure 1 from December 1925 to July 2003. The calibration uses a discounted WSAI growth rate set close to the empirical findings of Dimson, Marsh \& Staunton (2002), such that $\eta_{i}=0.05$ with an initial value of $\bar{\alpha}_{0}^{i}=0.05$. A comparison of Figure 1 to the original WSAI data reveals that the GOP volatility implied by the SMMM realistically models the leverage effect first discussed in Black (1976). When the index moves up, the volatility moves down and vice-versa, which can be observed in formula (3.9) since the squared volatility of the GOP is proportional to the inverse of the GOP itself, and hence the WSAI.

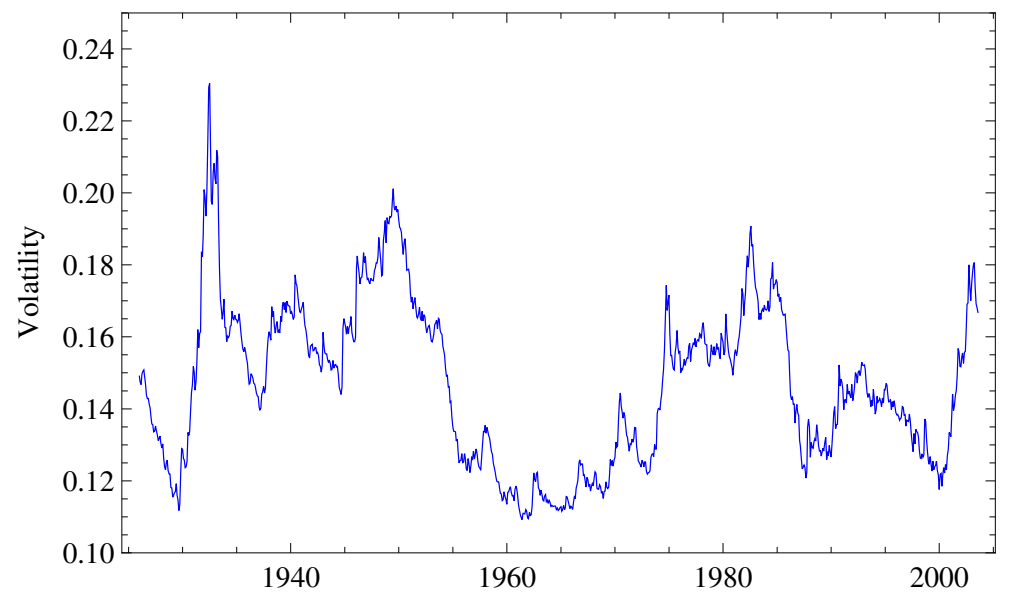

Figure 1: WSAI volatility $\left|\theta_{t}^{i}\right|$ in USD from 1925 to 2003 under the SMMM.

The GOP volatility in Figure 1 is the historical sample path implied by the SMMM. One way to understand the dynamics for the volatility of the GOP, is to define the squared volatility $v^{i}=\left\{v_{t}^{i}=\left|\theta_{t}^{i}\right|^{2}, t \in[0, T]\right\}, i \in\{0,1, \ldots, d\}$. Then by (3.2), (3.9) and the Itô formula, one obtains an SDE for the squared volatility of the GOP as

$$
d v_{t}^{i}=\eta_{i} v_{t}^{i} d t-\left(v_{t}^{i}\right)^{3 / 2} d W_{t}^{i}
$$

for $t \in[0, T]$ and $i \in\{0,1, \ldots, d\}$. This is a surprisingly simple stochastic volatility model. Because of the $3 / 2$ power appearing in the diffusion coefficient, such volatility dynamics have been referred to as a $3 / 2$ model. It was proposed in Platen (1997) and similar models were studied in Lewis (2000). We emphasize that there is only one Wiener process driving the dynamics of the GOP and its squared volatility. The resulting negative correlation between the two processes models the leverage effect observed in stock market indices.

Now consider the transition density function $p_{4}\left(\varphi_{t}^{i}, x_{t}^{i} ; \varphi_{\bar{T}}^{i}, x_{\bar{T}}^{i}\right)$ for a time transformed BESQ process $X^{i}=\left\{X_{\varphi_{t}^{i}}^{i}, \varphi_{t}^{i} \in\left[\varphi_{0}^{i}, \varphi_{\bar{T}}^{i}\right]\right\} i \in\{0,1, \ldots, d\}$, of dimension four, to move from $x_{t}^{i}=X_{\varphi_{t}^{i}}^{i}$ at time $\varphi_{t}^{i}$ to $x_{\bar{T}}^{i}=X_{\varphi_{\bar{T}}^{i}}^{i}$ at time $\varphi_{\bar{T}}^{i}$. It is derived in 
Revuz \& Yor (1999) in the form

$$
p_{4}\left(\varphi_{t}^{i}, x_{t}^{i} ; \varphi_{\bar{T}}^{i}, x_{\bar{T}}^{i}\right)=\frac{1}{2\left(\varphi_{\bar{T}}^{i}-\varphi_{t}^{i}\right)}\left(\frac{x_{\bar{T}}^{i}}{x_{t}^{i}}\right)^{\frac{1}{2}} \exp \left\{-\frac{x_{t}^{i}+x_{\bar{T}}^{i}}{2\left(\varphi_{\bar{T}}^{i}-\varphi_{t}^{i}\right)}\right\} I_{1}\left(\frac{\sqrt{x_{t}^{i} x_{\bar{T}}^{i}}}{\varphi_{\bar{T}}^{i}-\varphi_{t}^{i}}\right)
$$

for $\varphi_{t}^{i} \in\left[\varphi_{0}^{i}, \varphi_{\bar{T}}^{i}\right], i \in\{0,1, \ldots, d\}$ with the definition of the modified Bessel function of the first kind $I_{\ell}(\cdot)$ for index $\ell$, given by relation (A.4) in Appendix A.

\section{Non-Central Chi-Square with Zero Degrees of Freedom}

The transition density of a BESQ process is related to central and non-central chi-square random variables, the latter of which are summarised in Appendix A. A central chi-square random variable with $\nu \in[0, \infty)$ degrees of freedom has a distribution function denoted as $\chi^{2}(\cdot ; \nu)$. The corresponding non-central chi-square distributed random variable with $\nu$ degrees of freedom and non-centrality parameter $\lambda \in(0, \infty)$ has probability density $p_{\chi^{2}}(\cdot ; \nu, \lambda)$ and its distribution function is denoted $\chi^{2}(\cdot ; \nu, \lambda)$. Of particular interest are non-central chi-square distributed random variables with zero degrees of freedom, first studied in Torgerson (1972) and subsequently by Siegel (1979) and Jones (1987). Making the substitution of $\nu=0$ into the definition of the non-central chi-square distribution function (A.2) leads to

$$
\begin{aligned}
\chi^{2}(u ; 0, \lambda) & =\sum_{\ell=0}^{\infty} \frac{\exp \left\{-\frac{\lambda}{2}\right\}\left(\frac{\lambda}{2}\right)^{\ell}}{\ell !} \chi^{2}(u ; 2 \ell) \\
& =\exp \left\{-\frac{\lambda}{2}\right\}+\sum_{\ell=1}^{\infty} \frac{\exp \left\{-\frac{\lambda}{2}\right\}\left(\frac{\lambda}{2}\right)^{\ell}}{\ell !} \chi^{2}(u ; 2 \ell)
\end{aligned}
$$

for $u \geq 0$ and $\lambda>0$. As in Siegel (1979), we apply the convention that the case of $\ell=0$ corresponds to the outcome $u=0$ with probability $p_{\chi^{2}}(u ; 0, \lambda)=$ $\exp \{-\lambda / 2\}$. One has to be careful not to confuse this with the fact that $\chi^{2}(u ; 0)=$ 1 for all $u>0$. Thus from the first line in $(3.12), \chi^{2}(u ; 0, \lambda)$ is a mixture of the distributions $0, \chi^{2}(u ; 2), \chi^{2}(u ; 4), \ldots$ with Poisson weights. This means that the non-central chi-square distribution with zero degrees of freedom has a discrete component at zero and a continuous component for all $u>0$. This property actually holds for all non-central chi-square random variables with degrees of freedom $\nu \leq 2$. The analogy to BESQ processes is obvious since for $\nu>2$ the process remains strictly positive, but for $\nu \leq 2$ the boundary condition at zero must be specified, which is usually absorption. Although the distribution exists the corresponding probability density is improper since it does not sum to unity, as shown here

$$
\int_{0}^{\infty} p_{\chi^{2}}(x ; 0, \lambda) d x=1-\exp \{-\lambda / 2\}<1
$$


for $\lambda>0$. This result reflects the existence of positive probability mass at zero. It is interesting to examine graphical illustrations of the probability density $p_{\chi^{2}}(x ; 0, \lambda)$, as provided in Figure 2 below. The panel on the left-hand-side provides the density in the case with non-centrality parameter $\lambda=2$, whilst the right-hand-side panel illustrates the density for $\lambda=5$. One can easily observe the mixture of discrete and continuous components of the non-central chi-square probability density with zero degrees of freedom in either of these two charts.
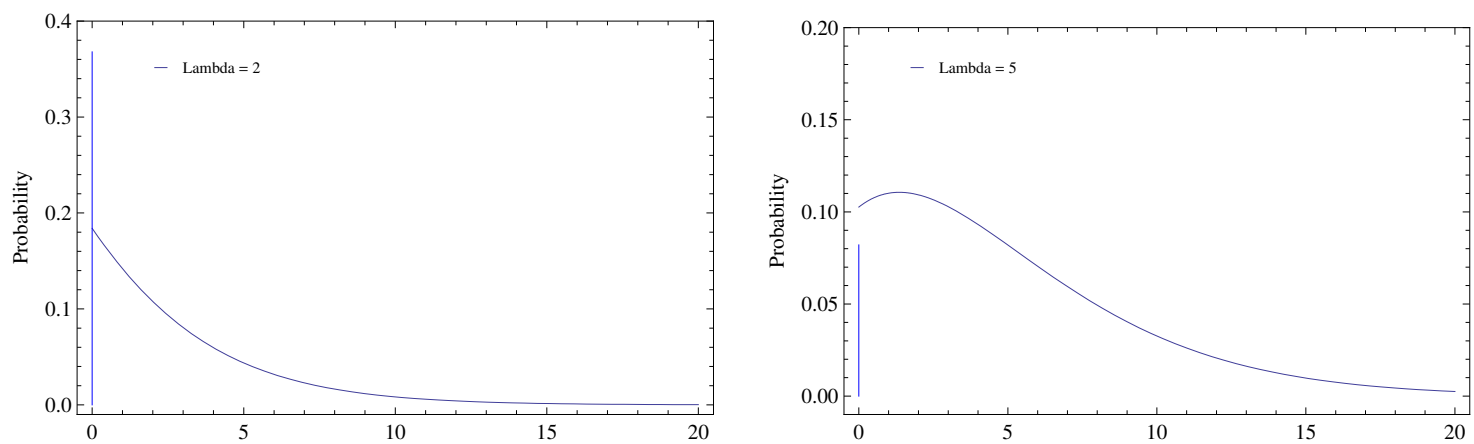

Figure 2: $p_{\chi^{2}}(\cdot ; 0, \lambda)$ and absorption probability, for $\lambda=2$ and $\lambda=5$ respectively.

\section{Interest Rate Term Structure}

The first investigation of the interest rate term structure within the benchmark framework was in Platen (2002). In that research it was shown that restrictions exist on the drift of the forward rate equation under the real-world measure that are analogous to those obtained for the candidate risk-neutral measure in Heath, Jarrow \& Morton (1992). Next Platen (2005a) provided the first model for the interest rate term structure in the context of the SMMM.

Zero coupon bonds are fundamental securities in financial markets. We formally define zero-coupon bonds in the benchmark framework as follows. The price of a zero-coupon bond $P^{i}(t, \bar{T})$ in the $i$ th currency at time $t$ with fixed maturity $\bar{T} \in[0, T]$ is defined as the time $t$ of one unit of the $i$ th currency, and is found using the real-world pricing formula (2.7) as

$$
P^{i}(t, \bar{T})=E\left[\frac{S_{t}^{i, \delta_{*}}}{S_{\bar{T}}^{i, \delta_{*}}} \mid \mathcal{A}_{t}\right]
$$

for $t \in[0, \bar{T}]$ and $i \in\{0,1, \ldots, d\}$. Note that $P^{i}(\bar{T}, \bar{T})=1$. Throughout this paper we will assume that the driving processes of the $i$ th short rate $r^{i}$ and the discounted GOP $\bar{S}^{i, \delta_{*}}$ are independent. This assumption allows us to characterise 
the zero-coupon bond price $P^{i}(t, \bar{T})$ in the following multiplicative way

$$
P^{i}(t, \bar{T})=E\left[\frac{\bar{S}_{t}^{i, \delta_{*}}}{\bar{S}_{\bar{T}}^{i, \delta_{*}}} \frac{B_{t}^{i}}{B_{\bar{T}}^{i}} \mid \mathcal{A}_{t}\right]=M_{\bar{T}}^{i}(t) G_{\bar{T}}^{i}(t)
$$

where the discounted GOP contribution to the zero-coupon bond price is

$$
M_{\bar{T}}^{i}(t)=E\left[\frac{\bar{S}_{t}^{i, \delta_{*}}}{\bar{S}_{\bar{T}}^{i, \delta_{*}}} \mid \mathcal{A}_{t}\right]=E\left[\frac{\Lambda_{\bar{T}}^{i, \theta}}{\Lambda_{t}^{i, \theta}} \mid \mathcal{A}_{t}\right]
$$

and the short rate contribution to the bond price is

$$
G_{\bar{T}}^{i}(t)=E\left[\frac{B_{t}^{i}}{B_{\bar{T}}^{i}} \mid \mathcal{A}_{t}\right]
$$

for $t \in[0, \bar{T}]$ and $i \in\{0,1, \ldots, d\}$. An example where the discounted GOP and the short rate are assumed to be independent, is studied in Miller \& Platen (2005). Another relevant example is when the short rate is assumed to be deterministic and constant, which we do for the remainder of this paper.

The expression in (4.3) referred to as the discounted GOP contribution to the zero-coupon bond price is quite an important quantity. The second equality in (4.3) reminds us that it can also be interpreted as the expected value of the RadonNikodym derivative for the candidate risk-neutral measure. An equivalent riskneutral probability measure will exist if and only if $\Lambda^{i, \theta}$ is an $(\underline{\mathcal{A}}, P)$-martingale and hence $M_{\bar{T}}^{i}(t)=1$ in (4.3) for all $t \in[0, \bar{T}]$ and $i \in\{0,1, \ldots, d\}$.

In Platen (2005a) it was shown tha the real-world price of a zero-coupon bond $P^{i}(t, \bar{T})$ calculated at time $t$ with maturity date $\bar{T} \in[0, T]$ under the given SMMM equals

$$
P^{i}(t, \bar{T})=\exp \left\{-r_{i}(\bar{T}-t)\right\}\left(1-\exp \left\{-\lambda_{\bar{T}}^{i} / 2\right\}\right)
$$

where

$$
\lambda_{\bar{T}}^{i}=\frac{4 \eta_{i}}{\left|\theta_{t}^{i}\right|^{2}\left(\exp \left\{\eta_{i}(\bar{T}-t)\right\}-1\right)}
$$

for $t \in[0, \bar{T}]$ and $i \in\{0,1, \ldots, d\}$.

Given that the short rate is assumed to be constant, it is trivial to observe that the zero-coupon bond price decomposition (4.2) applies. Hence it can be deduced 
from (4.3), (4.4) and (4.5) that for the SMMM

$$
\begin{aligned}
& G_{\bar{T}}^{i}(t)=E\left[\frac{B_{t}^{i}}{B_{\bar{T}}^{i}} \mid \mathcal{A}_{t}\right]=\exp \left\{-r_{i}(\bar{T}-t)\right\} \\
& M_{\bar{T}}^{i}(t)=E\left[\frac{\bar{S}_{t}^{i, \delta_{*}}}{\bar{S}_{\bar{T}}^{i, \delta_{*}}} \mid \mathcal{A}_{t}\right]=1-\exp \left\{-\lambda_{\bar{T}}^{i} / 2\right\}
\end{aligned}
$$

for $t \in[0, \bar{T}]$ and $i \in\{0,1, \ldots, d\}$. This relatively straightforward calculation provides some important insights into the SMMM that hitherto have not been discussed. Since the non-centrality parameter in (4.6) will, in general, be strictly positive, then (4.8) will be less than or equal to unity. Therefore we conclude that under the SMMM, the candidate Radon-Nikodym derivative process $(2.9)$ with volatility defined by $(3.9)$ is a strict $(\underline{\mathcal{A}}, P)$-supermartingale. Thus in determining the real-world price of a zero-coupon bond, we have confirmed the earlier discussion that a key assumption of classical risk-neutral pricing, namely that of Girsanov's Theorem, is not satisfied. Under the Fundamental Theorem of Asset Pricing of Delbaen \& Schachermayer (1994), this strict supermartingale property would exclude the SMMM as a model. However, under the benchmark approach, the SMMM is not excluded. In Figure 3 below we plot $M_{\bar{T}}^{i}(0)$ for the two different maturity ranges of: $\bar{T}=30$ years and $\bar{T}=100$ years. The remaining parameters used were: $\left|\theta_{0}^{i}\right|=0.25$; and $\eta_{i}=0.05$. Observe that $M_{\bar{T}}^{i}(0)$ appears to be a martingale for at least the first five years, but then it gradually decays to zero. The strict supermartingale property of $M_{\bar{T}}^{i}(0)$ has important implications for the pricing of long-term financial contracts. Heuristically, the two plots of Figure 3 show that standard risk-neutral pricing may be reasonably accurate in the short term, say up to five years, however financial instruments with a maturity beyond this initial period may be mispriced.
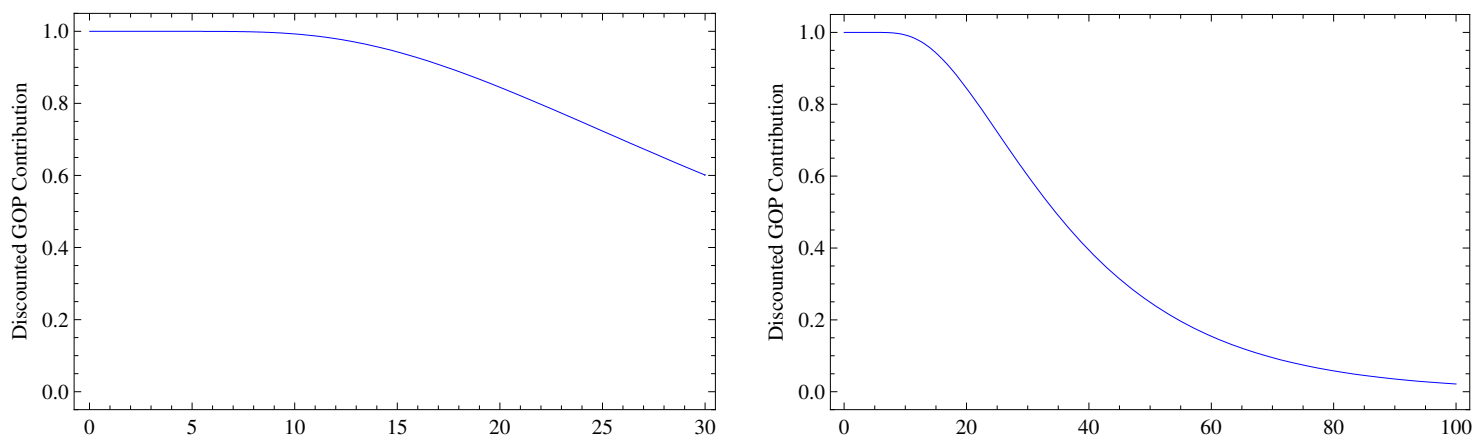

Figure 3: $M_{\bar{T}}^{i}(0)$ for $\bar{T} \in[0,30]$ and $\bar{T} \in[0,100]$ under the SMMM.

The second important observation is that the discounted GOP contribution to the bond price exactly matches the integral of the non-central chi-square random variable with zero degrees of freedom (3.13), and not by chance. Application of the results of Yor (1992) to (4.8) leads to an alternative expression for $M_{\bar{T}}^{i}(t)$ in 
the form

$$
M_{\bar{T}}^{i}(t)=\int_{0}^{\infty} \frac{x_{t}^{i}}{x_{\bar{T}}^{i}} p_{4}\left(\varphi_{t}^{i}, x_{t}^{i} ; \varphi_{\bar{T}}^{i}, x_{\bar{T}}^{i}\right) d x_{\bar{T}}^{i}=\int_{0}^{\infty} \tilde{p}_{0}\left(\varphi_{t}^{i}, x_{t}^{i} ; \varphi_{\bar{T}}^{i}, x_{\bar{T}}^{i}\right) d x_{\bar{T}}^{i}
$$

for $\varphi_{t}^{i} \in\left[\varphi_{0}^{i}, \varphi_{\bar{T}}^{i}\right]$ and $i \in\{0,1, \ldots, d\}$. Here $\tilde{p}_{0}\left(\varphi_{t}^{i}, x_{t}^{i} ; \varphi_{\bar{T}}^{i}, x_{\bar{T}}^{i}\right)$ represents the transition density of a BESQ process with dimension zero, where zero is an absorbing boundary. Therefore one observes that the law of the inverse of a BESQ process of dimension four is related to the law of a BESQ process with dimension zero. Furthermore, the right-hand-side of (4.9) is identical to the integral of the non-central chi-square probability density with zero degrees of freedom, given in (3.13). Recalling that the exponential term in (3.13) corresponds to the discrete component of the probability density at zero, we conclude that this exponential term represents the probability of absorption of a BESQ process of dimension zero at zero. Therefore any financial contract with a non-zero payoff at zero, such as a zero-coupon bond or a put option, must take into account this absorption probability. The benchmark approach accurately reflects this feature of the SMMM. On the other hand, the putative risk-neutral approach does not account for this possibility, irrespective of the underlying model assumptions. As an example, for the case under consideration, the putative risk-neutral zero-coupon bond price $P^{i, \theta}(t, \bar{T})$ will equal the short rate contribution to the zero-coupon bond price (4.7), hence $P^{i, \theta}(t, \bar{T})=G_{\bar{T}}^{i}(t)$ for all $\bar{T} \in[0, T]$ and $i \in\{0,1, \ldots, d\}$.

Given zero-coupon bond prices we can also calculate the instantaneous forward rate $f^{i}(t, \bar{T})$ at time $t$ for the maturity date $\bar{T} \in[0, T]$ and $i$ th currency, expressed as

$$
f^{i}(t, \bar{T})=-\frac{\partial}{\partial \bar{T}} \ln \left[P^{i}(t, \bar{T})\right]
$$

for all $t \in[0, \bar{T}]$ and $i \in\{0,1, \ldots, d\}$. Therefore, in the case when the zero-coupon bond price can be decomposed into the product in (4.2), then the forward rate (4.10) takes the form

$$
f^{i}(t, \bar{T})=m_{\bar{T}}^{i}(t)+g_{\bar{T}}^{i}(t)
$$

where the discounted GOP contribution to the forward rate is

$$
m_{\bar{T}}^{i}(t)=-\frac{\partial}{\partial \bar{T}} \ln \left[M_{\bar{T}}^{i}(t)\right]
$$

and the short rate contribution to the forward rate is

$$
g_{\bar{T}}^{i}(t)=-\frac{\partial}{\partial \bar{T}} \ln \left[G_{\bar{T}}^{i}(t)\right]
$$

for $t \in[0, \bar{T}]$ and $i \in\{0,1, \ldots, d\}$.

As a matter of completeness we also discuss instantaneous forward rates under 
the SMMM. Of course, the short rate contribution to the forward rate, defined by (4.13), is the short rate itself. Discussion was previously provided on the discounted GOP contribution to the forward rate in both Platen (2005a) and Miller \& Platen (2005). We provide a slightly different representation via (4.12) and (4.8) in the form

$$
m_{\bar{T}}^{i}(t)=\frac{\left(\lambda_{\bar{T}}^{i} / 2\right)^{2}\left|\theta_{t}^{i}\right|^{2} \exp \left\{\eta_{i}(\bar{T}-t)-\lambda_{\bar{T}}^{i} / 2\right\}}{2\left(1-\exp \left\{\lambda_{\bar{T}}^{i} / 2\right\}\right)}
$$

for all $t \in[0, \bar{T}]$ and $i \in\{0,1, \ldots, d\}$. Thus one observes that the volatility of the GOP is a key input to the discounted GOP contribution to the forward rate. In fact, a directional relationship exists between medium- and long-term forward rates and the volatility of the GOP that is simple, intuitive and economically sensible. Here the volatility of the GOP $\left|\theta_{t}^{i}\right|$ can be interpreted as the general level of uncertainty of the economy. A certain level of volatility in financial markets, and in the economy in general, is natural and healthy. However, when uncertainty increases beyond a particular threshold, the 'risk premium' in interest rate yields increases as compensation for this extra risk. This directional relationship is displayed for $m_{\bar{T}}^{i}(0)$ in Figure 4 below. The remaining input parameters used in this chart were: $t=0 ; \eta_{i}=0.05$; and $r_{i}=0.05$.

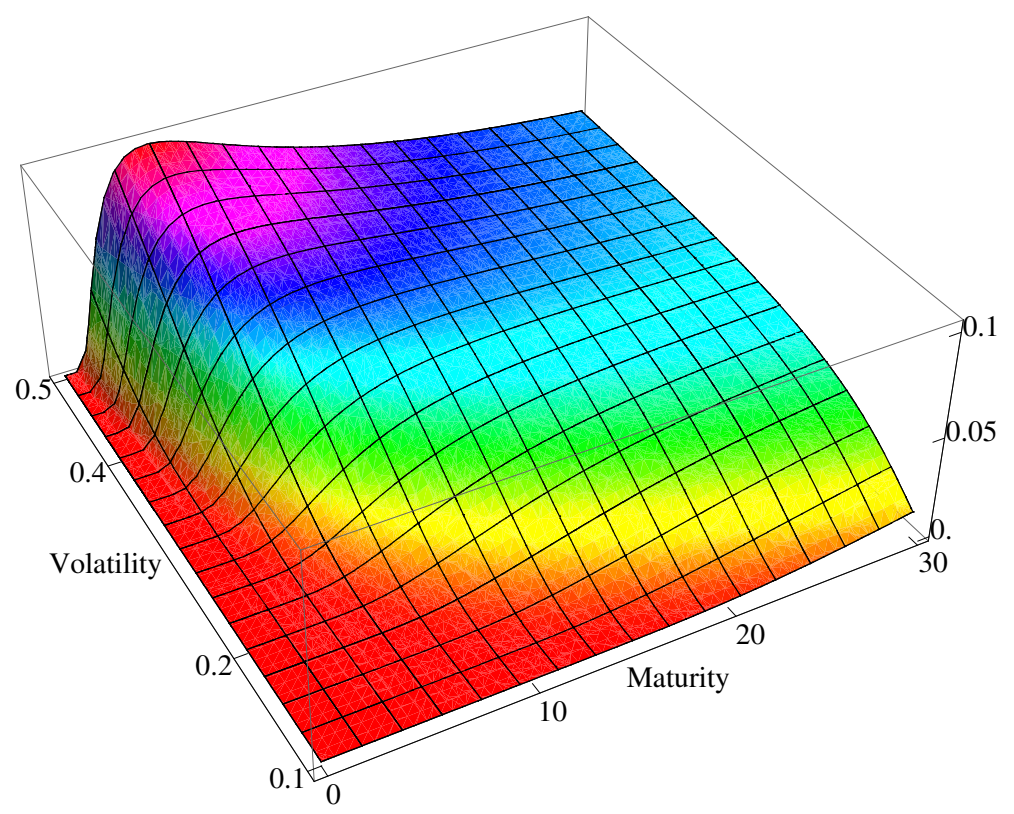

Figure 4: $m_{\bar{T}}^{i}(0)$ for $\left|\theta_{0}^{i}\right| \in[0.05,0.50]$ and $\bar{T} \in[0,30]$ under the SMMM. 


\section{Options on the GOP}

Numerical techniques have previously been employed to solve the problem of calculating real-world prices for options on the GOP under the given SMMM. Most notably, Heath \& Platen (2002) used numerical integration as an approach to this problem. However, analytic results are available, as we prove below. In addition to reducing computation time, the derived analytic formulae expressed in terms of non-central chi-square distribution functions, provide additional insights into the pricing and hedging of these options. For example, as in the case for the zero-coupon bond price, we find that the non-central chi-square distribution with zero degrees of freedom plays an interesting role in the final results. The proof of the following call and put option pricing formulae are given in Appendix B.

Lemma 5.1 The real-world prices of call and put options on the GOP at time $t$ with expiry date $\bar{T} \in[0, T]$ and strike price $K$ under the given SMMM are calculated as

$$
\begin{aligned}
& c_{\bar{T}, K, S^{i, \delta_{*}}}^{i}(t)=+S_{t}^{i, \delta_{*}}\left[1-\chi^{2}\left(\stackrel{*}{u}_{\bar{T}}^{i} ; 4, \lambda_{\bar{T}}^{i}\right)\right]-K e^{-r_{i}(\bar{T}-t)}\left[1-\chi^{2}\left(\stackrel{*}{u}_{\bar{T}}^{i} ; 0, \lambda_{\bar{T}}^{i}\right)\right] \\
& p_{\bar{T}, K, S^{i, \delta_{*}}}^{i}(t)=-S_{t}^{i, \delta_{*}} \chi^{2}\left(\stackrel{*}{u}_{\bar{T}}^{i} ; 4, \lambda_{\bar{T}}^{i}\right)+K e^{-r_{i}(\bar{T}-t)}\left[\chi^{2}\left(\stackrel{*}{u}_{\bar{T}}^{i} ; 0, \lambda_{\bar{T}}^{i}\right)-e^{-\lambda_{\bar{T}}^{i} / 2}\right]
\end{aligned}
$$

where

$$
\stackrel{*}{\bar{T}}_{\bar{T}}^{i}=\frac{4 \eta_{i} \exp \left\{-r_{i}(\bar{T}-t)\right\}}{\left|\theta_{t}^{i}\right|^{2}\left(\exp \left\{\eta_{i}(\bar{T}-t)\right\}-1\right)\left(S_{t}^{i, \delta_{*}} / K\right)}
$$

for $t \in[0, \bar{T}]$ and $i \in\{0,1, \ldots, d\}$ using notation (4.6).

The first conclusion from Lemma 5.1 is that the general put-call parity relationship for options on the GOP is found to be

$$
c_{\bar{T}, K, S^{i, \delta_{*}}}^{i}(t)+K P^{i}(t, \bar{T})=p_{\bar{T}, K, S^{i, \delta_{*}}}^{i}(t)+S_{t}^{i, \delta_{*}}
$$

for $t \in[0, \bar{T}]$ and $i \in\{0,1, \ldots, d\}$. Note that the real-world zero-coupon bond price given in (4.5) is necessary to achieve put-call parity. If one were to use the putative risk-neutral bond price of $P^{i, \theta}(t, \bar{T})=G_{\bar{T}}^{i}(t)$, then put-call parity would not hold.

Further examination of Lemma 5.1 reveals that the real-world price of an SMMM put option on the GOP contains an exponential term identical to that appearing within the zero-coupon bond price. These exponential terms are identical because they have the same origin. We can even be more specific and isolate the origin of exponential term to the bond binary or 'cash or nothing' component of the option on the GOP, which we denote by $B_{\bar{T}, K, S^{i, \delta_{*}}}^{i,+}(t)$ for a call and by $B_{\bar{T}, K, S^{i, \delta_{*}}}^{i,-}(t)$ for a put. Under the SMMM, these are essentially the expected value of the inverse 
of a BESQ process of dimension four, subject to certain boundary conditions. Application of the results of Yor (1992) allows us to rewrite these expressions in an analogous way to (4.9) as

$$
\begin{aligned}
& B_{\bar{T}, K, S^{i, \delta_{*}}}^{i,-}(t)=\frac{B_{t}^{i}}{B_{\bar{T}}^{i}} \int_{0}^{\frac{K}{B_{\bar{T}}^{i}}} \tilde{p}_{0}\left(\varphi_{t}^{i}, x_{t}^{i} ; \varphi_{\bar{T}}^{i}, x_{\bar{T}}^{i}\right) d x_{\bar{T}}^{i} \\
& B_{\bar{T}, K, S^{i, \delta_{*}}}^{i,+}(t)=\frac{B_{t}^{i}}{B_{\bar{T}}^{i}} \int_{\frac{K}{B_{\bar{T}}^{i}}}^{\infty} \tilde{p}_{0}\left(\varphi_{t}^{i}, x_{t}^{i} ; \varphi_{\bar{T}}^{i}, x_{\bar{T}}^{i}\right) d x_{\bar{T}}^{i}
\end{aligned}
$$

for $t \in[0, \bar{T}]$ and $i \in\{0,1, \ldots, d\}$. Here we see explicitly that a bond binary option on the GOP can be interpreted as the integrated probability density of a BESQ process of dimension zero, scaled by a ratio of savings accounts. Therefore the bond binary put option (5.5) involves evaluation of a process with a positive probability of reaching zero. The result includes the abovementioned exponential term, representing the probability of reaching zero, multiplied by the payoff of the put option at zero. In contrast, the bond binary call option has zero payoff at all points below the discounted strike price, as seen in (5.6). Thus the exponential term representing the probability of reaching zero is not reflected in the real-world call option price.

Lemma 5.1 also enables us to illustrate clear differences between the SMMM and the classical model of Black \& Scholes (1973). The best way to view the differences between the two models is to examine the implied volatility term structure of the given SMMM. Therefore in Figure 5 we plot the implied volatilities for options on the GOP for four different maturity times $\bar{T} \in\{10,20,50,100\}$ years. The implied volatilities for call and put options are equal under the given SMMM only when the Black-Scholes short rate input is implied from the real-world zerocoupon bond price given in (4.5). The parameters used in all charts were: $t=0$; $S_{0}^{i, \delta_{*}}=2,000 ;\left|\theta_{0}^{i}\right|=0.25 ; \eta_{i}=0.05 ;$ and $r_{i}=0.05$. One observes a skew in the direction of the strike in all of the charts in Figure 5. The other feature of note within Figure 5 is that implied volatilities increase for a period of up to ten years, and thereafter decline systematically to a long-run level.

The advantage of the approach here is that options on the GOP are presented in the analytic form of $(5.1)-(5.2)$. This provides an explicit understanding of the distributional properties underlying the SMMM. Furthermore, market practitioners are more receptive to analytic formulae similar to the standard Black-Scholes formulae than numerical techniques. Another advantage of analytic formulae is the direct availability of sensitivities with high accuracy, which are essential for hedging. However, we should comment on the numerical routines used in these analytic formulae defined in Lemma 5.1 and used to determine Figure 5. Many standard software packages, such as Mathematica, Matlab, Minitab, SPSS and SPlus, include built-in functions for the non-central chi-square distribution function (A.2). Notwithstanding, none of these in-built functions cater for the case 

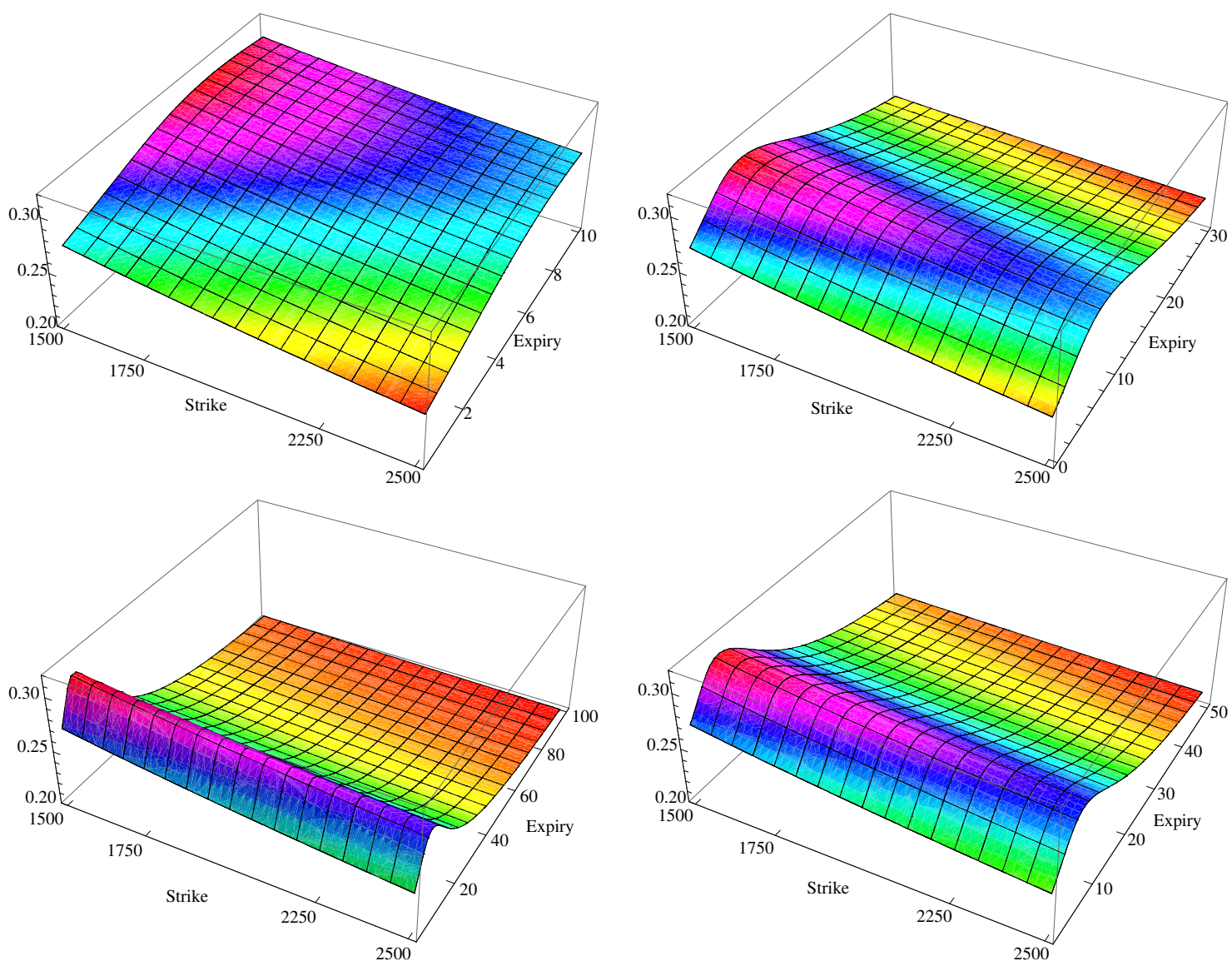

Figure 5: Implied volatilities for options on the GOP under the SMMM with $\bar{T} \in\{10,30,50,100\}$ years, clockwise respectively.

when the degrees of freedom is zero. Therefore we need to make use of alternative algorithms available from the literature. Of those available, we prefer and recommend the Ding (1992) implementation because it is intuitive, simple to implement, accurate and quite efficient under a variety of parameter constellations.

\section{Options on an Exchange Price}

The study of options on an exchange price under the SMMM is significantly more difficult than was the case for options on the GOP. Recall from (2.3) that we define an exchange price as the ratio of the GOP in two currency denominations. In order to maintain analytic formulae we make the simplifying assumption that different currency denominations of the discounted GOP are independent. Obviously, when the exchange price is a foreign currency exchange rate, especially a cross rate, this assumption may be too restrictive. However, there are many important cases when it is reasonable to impose such an assumption of independence. For example, an exchange price can also represent the price of a 
stock denominated in a particular currency. In cases where independence cannot be assumed we suggest using the numerical techniques described in Heath \& Platen (2005). In the following we use a probabilistic approach resulting in analytic formulae in terms of the doubly non-central beta distribution function $\mathcal{I}\left(\cdot ; \nu_{i}, \nu_{j} ; \lambda_{i}, \lambda_{j}\right)$ with the two degrees of freedom parameters $\nu_{i}, \nu_{j}$ and the two non-centrality parameters $\lambda_{i}, \lambda_{j}$, as defined in say Johnson, Kotz \& Balakrishnan (1995). We prove the following results in Appendix B.

Lemma 6.1 The real-world prices of call and put options on an exchange price $X^{i, j}$ in the ith currency denomination at time $t$ with expiry date $\bar{T} \in[0, T]$ and strike price $K$ under the given SMMM are

$$
\begin{aligned}
c_{\bar{T}, K, X^{i, j}}^{i}(t) & =+X_{t}^{i, j} e^{-r_{j}(\bar{T}-t)}\left[\mathcal{I}\left(\frac{1 / d_{i, j}}{1+1 / d_{i, j}} ; 0,4 ; \lambda_{\bar{T}}^{j}, \lambda_{\bar{T}}^{i}\right)-\exp \left\{-\frac{\lambda_{\bar{T}}^{j}}{2}\right\}\right] \\
& -K e^{-r_{i}(\bar{T}-t)}\left[1-\mathcal{I}\left(\frac{d_{i, j}}{1+d_{i, j}} ; 0,4 ; \lambda_{\bar{T}}^{i}, \lambda_{\bar{T}}^{j}\right)\right] \\
p_{\bar{T}, K, X^{i, j}}^{i}(t) & =-X_{t}^{i, j} e^{-r_{j}(\bar{T}-t)}\left[1-\mathcal{I}\left(\frac{1 / d_{i, j}}{1+1 / d_{i, j}} ;, 0 ; \lambda_{\bar{T}}^{j}, \lambda_{\bar{T}}^{i}\right)\right] \\
& +K e^{-r_{i}(\bar{T}-t)}\left[\mathcal{I}\left(\frac{d_{i, j}}{1+d_{i, j}} ; 0,4 ; \lambda_{\bar{T}}^{i}, \lambda_{\bar{T}}^{j}\right)-\exp \left\{-\frac{\lambda_{\bar{T}}^{i}}{2}\right\}\right]
\end{aligned}
$$

where

$$
d_{i, j}=\frac{\left|\theta_{t}^{j}\right|^{2}\left(\exp \left\{\eta_{j}(\bar{T}-t)\right\}-1\right) \eta_{i} \exp \left\{-r_{i}(\bar{T}-t)\right\}}{\left|\theta_{t}^{i}\right|^{2}\left(\exp \left\{\eta_{i}(\bar{T}-t)\right\}-1\right) \eta_{j} \exp \left\{-r_{j}(\bar{T}-t)\right\}\left(X_{t}^{i, j} / K\right)}
$$

for $t \in[0, \bar{T}]$ and $i, j \in\{0,1, \ldots, d\}$ and $\lambda_{\bar{T}}^{k}$ for $k \in\{i, j\}$ equates to (4.6).

The results from Lemma 6.1 prove that put-call parity only holds for an option on an exchange price when the real-world zero-coupon bond price (4.5) is used in the put-call parity relationship. Here, the strike price is discounted by the real-world zero-coupon bond in the $i$ th currency denomination, whilst the exchange price itself is discounted by the zero-coupon bond in the $j$ th denomination, such that

$$
c_{\bar{T}, K, X^{i, j}}^{i}(t)+K P^{i}(t, \bar{T})=p_{\bar{T}, K, X^{i, j}}^{i}(t)+X_{t}^{i, j} P^{j}(t, \bar{T})
$$

for $t \in[0, \bar{T}]$ and $i, j \in\{0,1, \ldots, d\}$.

We can also calculate implied volatility surfaces associated with call and put options on exchange prices under the SMMM, via the results of Lemma 6.1. Once again implied volatility surfaces provide the best method of comparison between the SMMM and the classical Black-Scholes model. Figure 6 below contains implied volatility surfaces for options on exchange prices for the four maturity times $\bar{T} \in\{10,30,50,100\}$ years. The other parameters used within the charts of Figure 
6 were: $t=0 ; X_{0}^{i, j}=1.0 ;\left|\theta_{0}^{i}\right|=\left|\theta_{0}^{j}\right|=0.25 ; \eta_{i}=\eta_{j}=0.05$; and $r_{i}=r_{j}=0.05$. As in previous sections, we include long-dated results to explore the effect of the strict supermartingale property of the putative Radon-Nikodym derivative. Indeed, we find that in the short term, out to say $\bar{T}=10$ years, the variation in implied volatility is relatively small, as observed in the top left-hand-side of Figure 6. As we move clockwise in Figure 6 to progressively longer time horizons, the implied volatility surface declines systematically as observed for options on the GOP. Also note that there is no skew visible in the implied volatilities for the chosen parameters.
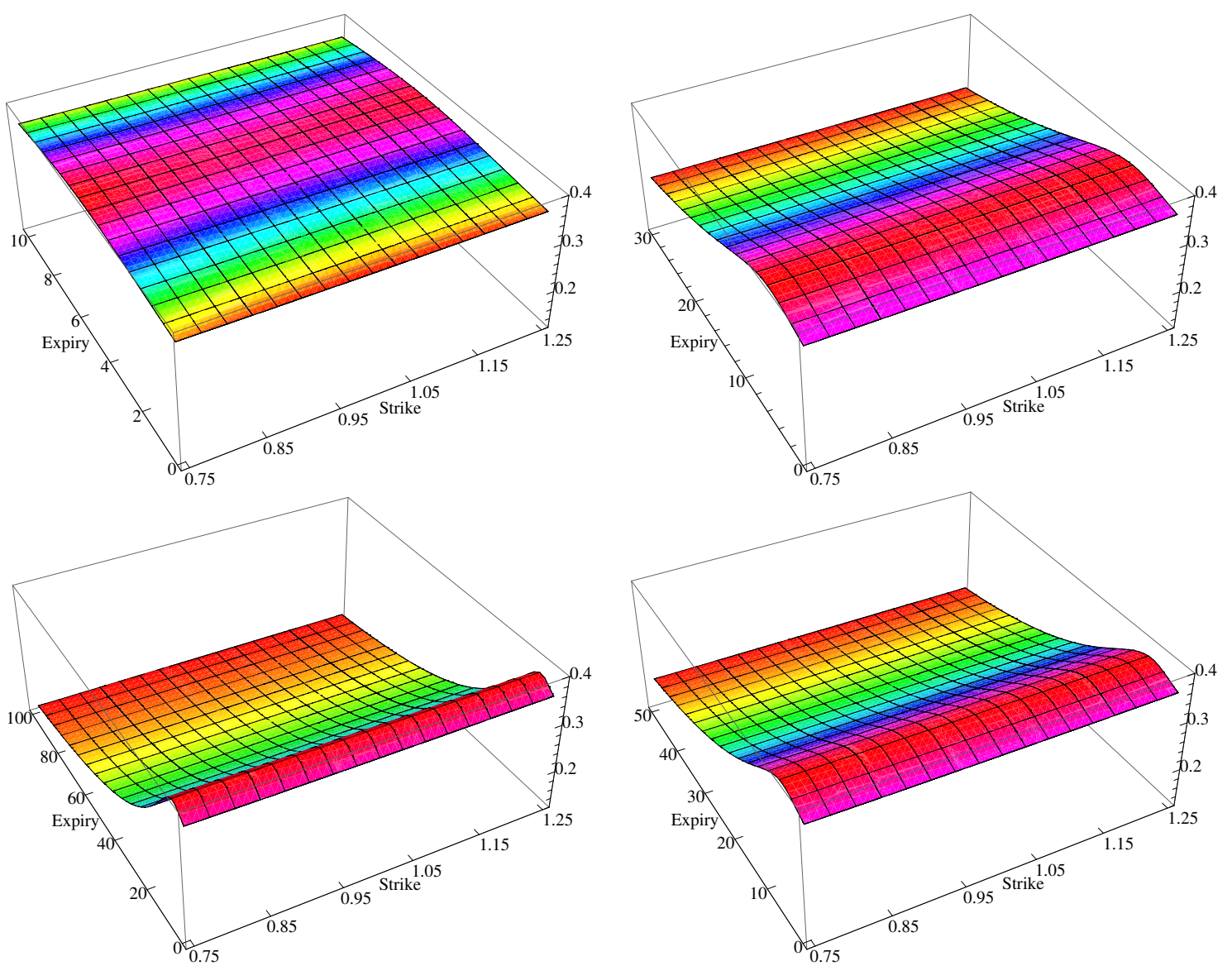

Figure 6: Implied volatilities for exchange price options under the SMMM with $\bar{T} \in\{10,30,50,100\}$ years, clockwise respectively.

Finally we recall the simple relationship between exchange prices and primary security accounts when the short rate is deterministic, given by (2.4). Therefore it is not surprising that real-world prices for options on primary security accounts take a similar form to those presented in Lemma 6.1, as can be seen in Hulley, Miller \& Platen (2005). 


\section{$7 \quad$ Interest Rate Options}

We now examine the real-world pricing of simple interest rate contingent claims under the SMMM. We maintain the assumption that the short rate is constant, hence $r_{t}^{i}=r_{i}$ for all $t \in[0, T]$ and $i \in\{0,1, \ldots, d\}$, and that the volatility of the GOP is defined by (3.9). As discussed in Section 4, these assumptions lead to a model of the interest rate term structure that can be decomposed into two components related to the discounted GOP and the short rate, as in (4.2). Furthermore, the restriction of a constant short rate limits the dynamics of forward rate behaviour for short- to medium-term maturities since all randomness is due to the discounted GOP, or equivalently the volatility of the GOP. A version of the SMMM with a stochastic short rate is examined in Miller \& Platen (2005).

It is obvious that the real-world zero-coupon bond price in (4.5) is bounded above by the hypothetical risk-neutral zero-coupon bond price. This upper bound for the bond price translates into a lower bound for the forward rate that equals the short rate. Therefore the SMMM forward rate will never fall below the short rate. We also know that the long-term equilibrium forward rate under the SMMM is asymptotically deterministic. In fact, Platen (2005a) showed that

$$
f^{i}(0, \infty)=r_{i}+\eta_{i}
$$

for $i \in\{0,1, \ldots, d\}$. Therefore the only variability for the interest rate term structure under the SMMM is found in medium- and long-term forward rates between the two anchor points of the initial short rate and the long-term asymptotic forward rate.

Even when the short rate is assumed to be constant, randomness due to the discounted GOP arises in the real-world pricing of zero-coupon bonds. Although such an interest rate term structure model is not very accurate for short-dated maturities, it deserves interest in that it allows us to study the impact of the discounted GOP on zero-coupon bonds, options on zero-coupon bonds and equivalently interest rate caps and floors. The following formulae are derived in Appendix B.

Lemma 7.1 The real-world prices of call and put options on a forward zerocoupon bond, $P^{i}(\bar{T}, T)$ with maturity $T \geq \bar{T}$ in the ith currency with strike price 
$K$ and the option expiry $\bar{T}$ under the given SMMM are

$$
\begin{aligned}
& \boldsymbol{z} \boldsymbol{b} \boldsymbol{c}_{\bar{T}, T, K}^{i}(t)=\left[+e^{-r_{i}(T-t)}-K e^{-r_{i}(\bar{T}-t)}\right]\left[1-\chi^{2}\left(\stackrel{*}{p}_{i} ; 0, \lambda_{\bar{T}}^{i}\right)\right] \\
& -e^{-r_{i}(T-t)} e^{-\frac{\lambda_{T}^{i}}{2}}\left[1-\chi^{2}\left(\hat{p}_{i} ; 0, \hat{\lambda}_{\bar{T}}^{i}\right)\right] \\
& \boldsymbol{z} \boldsymbol{b} \boldsymbol{p}_{\bar{T}, T, K}^{i}(t)= \begin{cases}-e^{-r_{i}(T-t)}\left[\chi^{2}\left(*_{i}^{*} ; 0, \lambda_{\bar{T}}^{i}\right)-e^{-\frac{\lambda_{T}^{i}}{2}} \chi^{2}\left(\hat{p}_{i} ; 0, \hat{\lambda}_{\bar{T}}^{i}\right)\right] \\
+K e^{-r_{i}(\bar{T}-t)}\left[\chi^{2}\left(*_{i}^{*} ; 0, \lambda_{\bar{T}}^{i}\right)-e^{-\frac{\lambda_{T}^{i}}{2}}\right] & \text { for } K<G_{T}^{i}(\bar{T}) \\
-P^{i}(t, T)+K P^{i}(t, \bar{T}) & \text { for } K \geq G_{T}^{i}(\bar{T})\end{cases}
\end{aligned}
$$

where

$$
\begin{aligned}
\lambda_{T}^{i} & =\frac{4 \eta_{i}}{\left|\theta_{t}^{i}\right|^{2}\left(\exp \left\{\eta_{i}(T-t)\right\}-1\right)} \\
\hat{\lambda}_{\bar{T}}^{i} & =\frac{4 \eta_{i}\left(\exp \left\{\eta_{i} T\right\}-\exp \left\{\eta_{i} \bar{T}\right\}\right)}{\left|\theta_{t}^{i}\right|^{2}\left(\exp \left\{\eta_{i}(\bar{T}-t)\right\}-1\right)\left(\exp \left\{\eta_{i}(T-t)\right\}-1\right)} \\
\dot{p}_{i}^{*} & =-\frac{2\left(\exp \left\{\eta_{i}(T-\bar{T})\right\}-1\right)}{1-\exp \left\{-\eta_{i}(\bar{T}-t)\right\}} \ln \left(\frac{G_{T}^{i}(\bar{T})-K}{G_{T}^{i}(\bar{T})}\right) \\
\hat{p}_{i} & =-\frac{2\left(\exp \left\{\eta_{i}(T-t)\right\}-1\right)}{\exp \left\{\eta_{i}(\bar{T}-t)\right\}-1} \ln \left(\frac{G_{T}^{i}(\bar{T})-K}{G_{T}^{i}(\bar{T})}\right)
\end{aligned}
$$

for $0 \leq t \leq \bar{T} \leq T$ and $i \in\{0,1, \ldots, d\}$ using notation (4.5)-(4.7).

One immediately observes from Lemma 7.1 that both the put option price (7.3) and the call option price (7.2) contain an exponential term, indicating once more the existence of a non-zero payoff at zero. This is consistent with the prior discussion on the pricing of zero-coupon bonds under the SMMM. Another interesting aspect of Lemma 7.1 is the separation of the put option price into two cases. The necessity of doing so, can be deduced from the structure of the logarithmic terms within relations $(7.6)-(7.7)$.

Lemma 7.1 can also be used to verify the put-call parity relationship for options on zero-coupon bonds, given in the general case as

$$
\mathbf{z b c}_{\bar{T}, T, K}^{i}(t)+K P^{i}(t, \bar{T})=\mathbf{z b p}_{\bar{T}, T, K}^{i}(t)+P^{i}(t, T)
$$

for $0 \leq t \leq \bar{T}<T$ and $i \in\{0,1, \ldots, d\}$. As in all previous cases in this paper, the real-world zero-coupon bond price (4.5) is necessary for put-call parity to hold.

The results from Lemma 7.1 are deliberately presented in a format facilitating comparison to prices for options on zero-coupon bonds under a putative riskneutral setting. In the case of a call option on a zero-coupon bond, the realworld SMMM price (7.2) will always be less than the corresponding hypothetical 
risk-neutral option price. However it is not possible to draw a similar definitive conclusion from a comparison between the real-world SMMM zero-coupon bond put option price (7.3) and a corresponding hypothetical risk-neutral price.

Now we examine the nature of interest rate caps and floors for real-world pricing under the SMMM as compared to the putative risk-neutral approach. We do so via the analysis of prototypical interest rate caplets and floorlets. This is possible since interest rate cap and floor behaviour can be examined via interest rate caplets and floorlets without loss of generality. The precise relationship between options on zero-coupon bonds, interest rate caps, caplets, floors and floorlets can be found in standard texts such as Brigo \& Mercurio (2006).

We investigate the essential characteristics of interest rate caplet and floorlet prices under the SMMM by plotting in Figure 7 at-the-money (ATM) caplet volatilities up to the four different expiry times of $\bar{T} \in\{10,30,50,100\}$ years. The remaining input parameters used in Figure 7 were: $t=0 ;\left|\theta_{0}^{i}\right|=0.25 ; \eta_{i}=0.05$; and $r_{i}=0.05$. One observes that short-term maturities in the volatility term structure are not influenced by the discounted GOP, which is consistent with our earlier results. Furthermore, the influence of the discounted GOP exerts on very long-dated maturities of the volatility term structure diminishes, as anticipated. The charts in Figure 7 also highlight that under the SMMM, although the caplet implied volatility term structure naturally exhibits a hump-shape, consistent with empirical data found in say Brigo \& Mercurio (2006), it fails to adequately capture short-dated implied volatility. In addition, the caplet implied volatility term structure is quite rigid, especially in terms of the location of the hump within the maturity spectrum. These latter restrictive features can be overcome in a number of ways, most importantly via the addition of a stochastic short rate, as discussed in Miller \& Platen (2005).

\section{Conclusion}

This paper derives a series of analytical formulae for various contingent claims using the stylised version of the minimal market model, where the discounted numeraire portfolio follows a time-transformed squared Bessel process. These new results include formulae for European call and put option prices on the GOP, options on exchange prices and options on zero-coupon bonds. The analytic formulae for options on zero-coupon bonds can be easily exploited to calculate prices for interest rate caps and floors. 

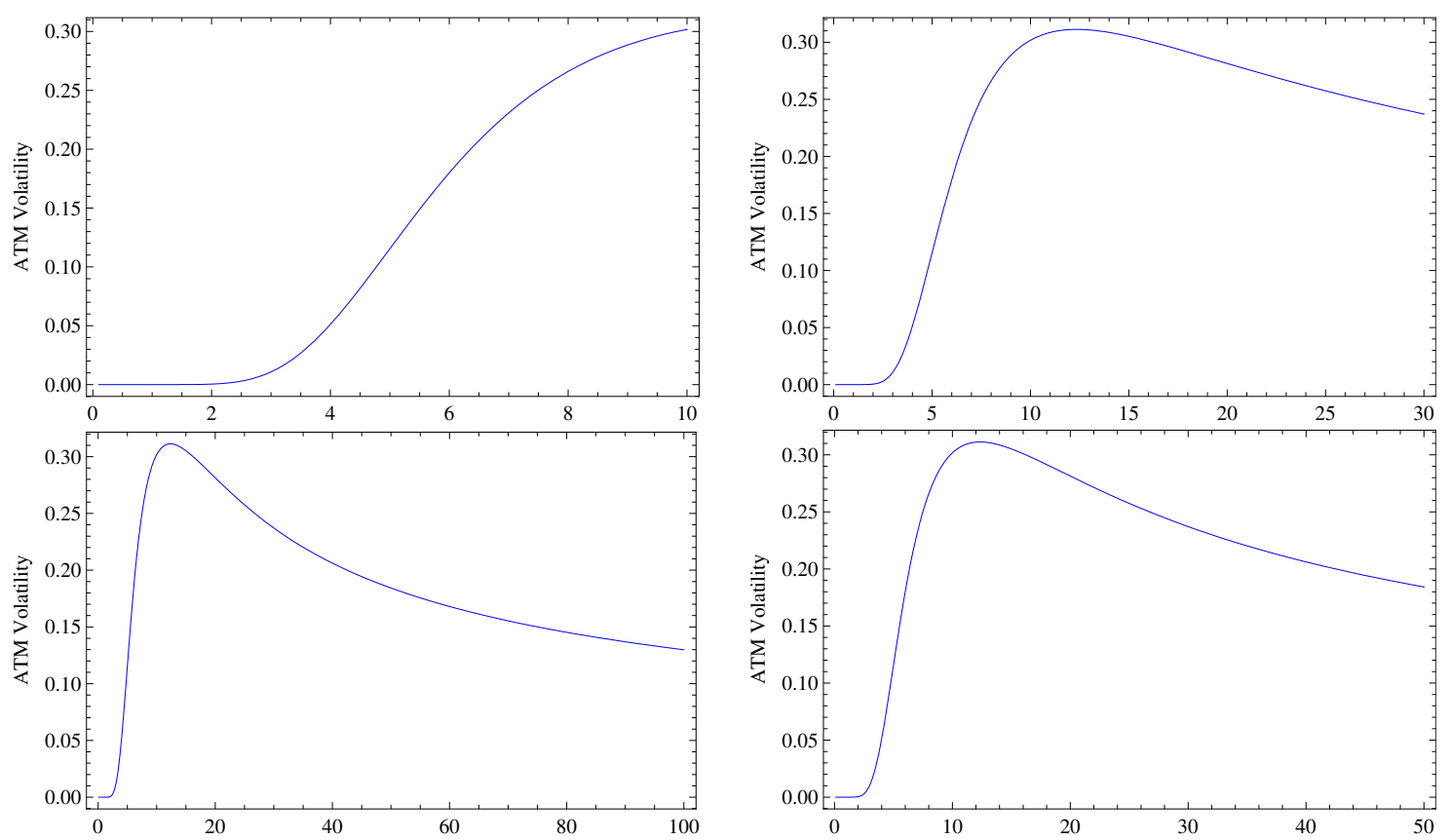

Figure 7: ATM interest rate caplet implied volatility term structure under the SMMM to $\bar{T} \in\{10,30,50,100\}$ years, clockwise.

\section{Appendix A}

Johnson, Kotz \& Balakrishnan (1995) define the density $p_{\chi^{2}}(x ; \nu, \lambda)$ and distribution function $\chi^{2}(u ; \nu, \lambda)$ for a non-central chi-square distributed random variable with $\nu$ degrees of freedom and non-centrality parameter $\lambda$ as

$$
\begin{aligned}
p_{\chi^{2}}(x ; \nu, \lambda) & =\sum_{\ell=0}^{\infty} \frac{\exp \left\{-\frac{\lambda}{2}\right\}\left(\frac{\lambda}{2}\right)^{\ell}}{\ell !} \frac{\left(\frac{x}{2}\right)^{\frac{\nu}{2}+\ell-1} \exp \left\{-\frac{x}{2}\right\}}{2 \Gamma\left(\frac{\nu}{2}+\ell\right)} \\
\chi^{2}(u ; \nu, \lambda) & =\int_{0}^{u} p_{\chi^{2}}(x ; \nu, \lambda) d x=\sum_{\ell=0}^{\infty} \frac{\exp \left\{-\frac{\lambda}{2}\right\}\left(\frac{\lambda}{2}\right)^{\ell}}{\ell !} \chi^{2}(u ; \nu+2 \ell)
\end{aligned}
$$

for $x>0, u \geq 0, \nu \geq 0$ and $\lambda \geq 0$ where $\chi^{2}(u ; \nu)$ represents the distribution function for a central chi-square distributed random variable with $\nu$ degrees of freedom. The non-central chi-square probability density (A.1) can be rewritten as

$$
p_{\chi^{2}}(x ; \nu, \lambda)=\frac{1}{2}\left(\frac{x}{\lambda}\right)^{\frac{\nu-2}{4}} \exp \left\{-\frac{\lambda+x}{2}\right\} I_{\frac{\nu-2}{2}}(\sqrt{\lambda x})
$$

for $x \geq 0, \nu \geq 0$ and $\lambda \geq 0$. This expression is identical in form to that of the transition density function of a squared Bessel process of general dimension $\nu>2$, as found in Revuz \& Yor (1999). Also note use of the modified Bessel 
function of the first kind $I_{d}(w)$, defined as

$$
I_{d}(w)=\left(\frac{w}{2}\right)^{d} \sum_{\ell=0}^{\infty} \frac{\left(\frac{w}{2}\right)^{2 \ell}}{\ell ! \Gamma(\ell+d+1)} .
$$

Comparison between transition density (3.11) with the probability density (A.1) reveals that equality between the two equations can be recovered by making the following transformations

$$
\begin{aligned}
& \lambda_{\bar{T}}^{i}=\frac{x_{t}^{i}}{\varphi_{\bar{T}}^{i}-\varphi_{t}^{i}}=\frac{X_{\varphi_{t}^{i}}^{i}}{\varphi_{\bar{T}}^{i}-\varphi_{t}^{i}}=\frac{\bar{S}_{t}^{i, \delta_{*}}}{\varphi_{\bar{T}}^{i}-\varphi_{t}^{i}} \\
& u_{\bar{T}}^{i}=\frac{x_{\bar{T}}^{i}}{\varphi_{\bar{T}}^{i}-\varphi_{t}^{i}}=\frac{X_{\varphi_{\bar{T}}^{i}}^{i}}{\varphi_{\bar{T}}^{i}-\varphi_{t}^{i}}=\frac{\bar{S}_{\bar{T}}^{i, \delta_{*}}}{\varphi_{\bar{T}}^{i}-\varphi_{t}^{i}}
\end{aligned}
$$

for $t \in[0, \bar{T}]$ and $i \in\{0,1, \ldots, d\}$.

\section{Appendix B}

Proof of Lemma 5.1: We apply the real-world pricing formula (2.7) to a call option on the GOP, thereby obtaining

$$
\begin{aligned}
c_{\bar{T}, K, S^{i, \delta_{*}}}^{i}(t) & =E\left[\frac{S_{t}^{i, \delta_{*}}}{S_{\bar{T}}^{i, \delta_{*}}}\left(S_{\bar{T}}^{i, \delta_{*}}-K\right)^{+} \mid \mathcal{A}_{t}\right] \\
& =S_{t}^{i, \delta_{*}} E\left[\mathbb{1}\left\{S_{\bar{T}}^{i, \delta_{*}} \geq K\right\} \mid \mathcal{A}_{t}\right]-K E\left[\frac{S_{t}^{i, \delta_{*}}}{S_{\bar{T}}^{i, \delta_{*}}} \mathbb{1}\left\{S_{\bar{T}}^{i, \delta_{*}} \geq K\right\} \mid \mathcal{A}_{t}\right] \\
& =A_{\bar{T}, K, S^{i, \delta_{*}}}^{i,+}(t)-K B_{\bar{T}, K, S^{i, \delta_{*}}}^{i,+}(t)
\end{aligned}
$$

for $t \in[0, \bar{T}]$ and $i \in\{0,1, \ldots, d\}$. Here we decompose the call option price into the two components of asset and bond binary call options in the notation of Buchen (2003). They are also referred to as 'asset or nothing' and 'cash or nothing' binary or digital options, respectively. If we set $x_{t}^{i}=X_{\varphi_{t}^{i}}^{i}$ and $x_{\bar{T}}^{i}=X_{\varphi_{\bar{T}}^{i}}^{i}$ and make use of (2.5), (3.6) and (3.11), then an asset binary call option on the GOP is

$$
A_{\bar{T}, K, S^{i, \delta_{*}}}^{i,+}(t)=S_{t}^{i, \delta_{*}} \int_{\frac{K}{B_{T}^{i}}}^{\infty} p_{4}\left(\varphi_{t}^{i}, x_{t}^{i} ; \varphi_{\bar{T}}^{i}, x_{\bar{T}}^{i}\right) d x_{\bar{T}}^{i}
$$

whilst the corresponding bond binary call option on the GOP is

$$
B_{\bar{T}, K, S^{i}, \delta_{*}}^{i,+}(t)=\frac{B_{t}^{i}}{B_{\bar{T}}^{i}} \int_{\frac{K}{B_{\bar{T}}^{i}}}^{\infty} \frac{x_{t}^{i}}{x_{\bar{T}}^{i}} p_{4}\left(\varphi_{t}^{i}, x_{t}^{i} ; \varphi_{\bar{T}}^{i}, x_{\bar{T}}^{i}\right) d x_{\bar{T}}^{i}
$$


for $\varphi_{t}^{i} \in\left[\varphi_{0}^{i}, \varphi_{\bar{T}}^{i}\right]$ and $i \in\{0,1, \ldots, d\}$. Substitution of (A.5)-(A.6) into the transition density and algebraic manipulation leads to the real-world price of a call option on the GOP under the SMMM as per (5.1). The corresponding realworld price of a put option on the GOP can be found using similar arguments or via the put-call parity relationship (5.4).

Proof of Lemma 6.1: Application of the real-world pricing formula (2.7) to a put option on an exchange price leads to

$$
\begin{aligned}
p_{\bar{T}, K, X^{i, j}}^{i}(t) & =E\left[\frac{S_{t}^{i, \delta_{*}}}{S_{\bar{T}}^{i, \delta_{*}}}\left(K-X_{\bar{T}}^{i, j}\right)^{+} \mid \mathcal{A}_{t}\right] \\
& =-X_{t}^{i, j} E\left[\frac{S_{t}^{j, \delta_{*}}}{S_{\bar{T}}^{j, \delta_{*}}} \mathbb{1}\left\{X_{\bar{T}}^{i, j}<K\right\} \mid \mathcal{A}_{t}\right]+K E\left[\frac{S_{t}^{i, \delta_{*}}}{S_{\bar{T}}^{i, \delta_{*}}} \mathbb{1}\left\{X_{\bar{T}}^{i, j}<K\right\} \mid \mathcal{A}_{t}\right] \\
& =-A_{\bar{T}, K, X^{i, j}}^{i,-}(t)+K B_{\bar{T}, K, X^{i, j}}^{i,-}(t)
\end{aligned}
$$

for $t \in[0, \bar{T}]$ and $i, j \in\{0,1, \ldots, d\}$. Thus the put option price can be represented by asset and bond binary put options in the notation of Buchen (2003). In the case of the SMMM, we combine (2.5) with (3.6) to yield an asset binary put option on an exchange price of

$$
A_{\bar{T}, K, X^{i, j}}^{i,-}(t)=X_{t}^{i, j} \frac{B_{t}^{j}}{B_{\bar{T}}^{j}} E\left[\frac{X_{\varphi_{t}^{j}}^{j}}{X_{\varphi_{\bar{T}}^{j}}^{j}} \mathbb{1}\left\{X_{\varphi_{\bar{T}}^{j}}^{j} \geq \frac{X_{\varphi_{\bar{T}}^{i}}^{i} B_{\bar{T}}^{i}}{K B_{\bar{T}}^{j}}\right\} \mid \mathcal{A}_{t}\right]
$$

whilst the corresponding bond binary option on an exchange price is

$$
B_{\bar{T}, K, X^{i, j}}^{i,-}(t)=\frac{B_{t}^{i}}{B_{\bar{T}}^{i}} E\left[\frac{X_{\varphi_{t}^{i}}^{i}}{X_{\varphi_{\bar{T}}^{i}}^{i}} \mathbb{1}\left\{X_{\varphi_{\bar{T}}^{j}}^{i}<\frac{K X_{\varphi_{\bar{T}}^{j}}^{j} B_{\bar{T}}^{j}}{B_{\bar{T}}^{i}}\right\} \mid \mathcal{A}_{t}\right]
$$

for $t \in[0, \bar{T}]$ and $i, j \in\{0,1, \ldots, d\}$. Next we invoke the assumption that different currency denominations of the GOP are independent. Hence the expectations in (B.6)-(B.7) can be written in terms of two transition densities in the form of (3.11). If we make the substitutions of $x_{t}^{k}=X_{\varphi_{t}^{k}}^{k}$ and $x_{\bar{T}}^{k}=X_{\varphi_{\bar{T}}^{k}}^{k}$ for $k \in\{i, j\}$, then we obtain the asset binary put option relation (B.6) as

$$
A_{\bar{T}, K, X^{i, j}}^{i,-}(t)=X_{t}^{i, j} \frac{B_{t}^{j}}{B_{\bar{T}}^{j}} \int_{0}^{\infty} \int_{\frac{x_{\bar{T}}^{i} B_{\bar{T}}^{i}}{K B_{\bar{T}}^{j}}}^{\infty} \frac{x_{t}^{j}}{x_{\bar{T}}^{j}} p_{4}\left(\varphi_{t}^{j}, x_{t}^{j} ; \varphi_{\bar{T}}^{j}, x_{\bar{T}}^{j}\right) p_{4}\left(\varphi_{t}^{i}, x_{t}^{i} ; \varphi_{\bar{T}}^{i}, x_{\bar{T}}^{i}\right) d x_{\bar{T}}^{j} d x_{\bar{T}}^{i}
$$


whilst the corresponding bond binary put option relation (B.7) becomes

$$
B_{\bar{T}, K, X^{i, j}}^{i,-}(t)=\frac{B_{t}^{i}}{B_{\bar{T}}^{i}} \int_{0}^{\infty} \int_{0}^{\frac{K x_{\bar{T}}^{j} B_{\bar{T}}^{j}}{B_{\bar{T}}^{i}}} \frac{x_{t}^{i}}{x_{\bar{T}}^{i}} p_{4}\left(\varphi_{t}^{i}, x_{t}^{i} ; \varphi_{\bar{T}}^{i}, x_{\bar{T}}^{i}\right) p_{4}\left(\varphi_{t}^{j}, x_{t}^{j} ; \varphi_{\bar{T}}^{j}, x_{\bar{T}}^{j}\right) d x_{\bar{T}}^{i} d x_{\bar{T}}^{j}
$$

for $t \in[0, \bar{T}]$ and $i, j \in\{0,1, \ldots, d\}$. Substitution of (A.5)-(A.6) in each currency denomination $i, j \in\{0,1, \ldots, d\}$ and a significant amount of algebra yield the real-world price of a put option on an exchange price under the SMMM as (6.2). The corresponding real-world price of a call option on an exchange price is found using similar reasoning or via the put-call parity relationship (6.4).

Proof of Lemma 7.1: We apply the real-world pricing formula (2.7) to a call option on a forward zero-coupon bond $P^{i}(\bar{T}, T)$ to obtain

$$
\begin{aligned}
c_{\bar{T}, K, P^{i}(\bar{T}, T)}^{i}(t) & =E\left[\frac{S_{t}^{i, \delta_{*}}}{S_{\bar{T}}^{i, \delta_{*}}}\left(P^{i}(\bar{T}, T)-K\right)^{+} \mid \mathcal{A}_{t}\right] \\
& =A_{\bar{T}, K, P^{i}(\bar{T}, T)}^{i,+}(t)-K B_{\bar{T}, K, P^{i}(\bar{T}, T)}^{i,+}(t)
\end{aligned}
$$

for $0 \leq t \leq \bar{T} \leq T$ and $i \in\{0,1, \ldots, d\}$. Again, it is instructive to decompose the call option price into constituent call asset and bond binary options. Bond binary options on zero-coupon bonds are similar in structure to bond binary options on the GOP, since we obtain

$$
B_{\bar{T}, K, P^{i}(\bar{T}, T)}^{i,+}(t)=E\left[\frac{S_{t}^{i, \delta_{*}}}{S_{\bar{T}}^{i, \delta_{*}}} \mathbb{1}_{\left\{P^{i}(\bar{T}, T) \geq K\right\}} \mid \mathcal{A}_{t}\right]=\frac{B_{t}^{i}}{B_{\bar{T}}^{i}} E\left[\frac{\bar{S}_{t}^{i, \delta_{*}}}{\bar{S}_{\bar{T}}^{i, \delta_{*}}} \mathbb{1}_{\left\{\bar{S}_{\bar{T}}^{i, \delta_{*}} \geq \bar{p}_{i}\right\}} \mid \mathcal{A}_{t}\right]
$$

where

$$
\bar{p}_{i}=-2\left(\varphi_{T}^{i}-\varphi_{\bar{T}}^{i}\right) \ln \left(\frac{G_{T}^{i}(\bar{T})-K}{G_{T}^{i}(\bar{T})}\right)
$$

for $0 \leq t \leq \bar{T} \leq T$ and $i \in\{0,1, \ldots, d\}$. The solution to (B.11) comes from the result for a bond binary call option on the GOP within the proof of Lemma 5.1.

The asset binary call option on a forward zero-coupon bond $P^{i}(\bar{T}, T)$ is found as

$$
\begin{aligned}
A_{\bar{T}, K, P^{i}(\bar{T}, T)}^{i,+}(t) & =E\left[\frac{S_{t}^{i, \delta_{*}}}{S_{\bar{T}}^{i, \delta_{*}}} P^{i}(\bar{T}, T) \mathbb{1}_{\left\{P^{i}(\bar{T}, T) \geq K\right\}} \mid \mathcal{A}_{t}\right] \\
& =G_{T}^{i}(\bar{T}) E\left[\frac{B_{t}^{i}}{B_{\bar{T}}^{i}} \frac{\bar{S}_{t}^{i, \delta_{*}}}{\bar{S}_{\bar{T}}^{i, \delta_{*}}} M_{T}^{i}(\bar{T}) \mathbb{1}_{\left\{\bar{S}_{\bar{T}}^{i, \delta_{*}} \geq \bar{p}_{i}\right\}} \mid \mathcal{A}_{t}\right]
\end{aligned}
$$


for $0 \leq t \leq \bar{T} \leq T$ and $i \in\{0,1, \ldots, d\}$ using (4.7), (4.8) and (B.12). Expanding (4.8) within expectation (B.13) leads to

$$
A_{\bar{T}, K, P^{i}(\bar{T}, T)}^{i,+}(t)=G_{T}^{i}(\bar{T})\left[B_{\bar{T}, K, P^{i}(\bar{T}, T)}^{i,+}(t)-\Xi_{\bar{T}, T, P^{i}(\bar{T}, T)}^{i,+}(t)\right]
$$

where

$$
\begin{aligned}
\Xi_{\bar{T}, T, P^{i}(\bar{T}, T)}^{i,+}(t) & =E\left[\frac{S_{t}^{i, \delta_{*}}}{S_{\bar{T}}^{i, \delta_{*}}} \exp \left\{-\frac{\bar{S}_{\bar{T}}^{i, \delta_{*}}}{2\left(\varphi_{T}^{i}-\varphi_{\bar{T}}^{i}\right)}\right\} \mathbb{1}_{\left\{\bar{S}_{\bar{T}}^{\left.i, \delta_{*} \geq \bar{p}_{i}\right\}}\right.} \mid \mathcal{A}_{t}\right] \\
& =\frac{B_{t}^{i}}{B_{T}^{i}} \int_{\bar{p}_{i}}^{\infty} \frac{x_{t}^{i}}{x_{\bar{T}}^{i}} \exp \left\{-\frac{x_{\bar{T}}^{i}}{2\left(\varphi_{T}^{i}-\varphi_{\bar{T}}^{i}\right)}\right\} p_{4}\left(\varphi_{t}^{i}, x_{t}^{i} ; \varphi_{\bar{T}}^{i}, x_{\bar{T}}^{i}\right) d x_{\bar{T}}^{i}
\end{aligned}
$$

for $0 \leq t \leq \bar{T} \leq T$ and $i \in\{0,1, \ldots, d\}$ using (B.11). We also introduce the auxiliary function $\Xi_{\bar{T}, T, P^{i}(\bar{T}, T)}^{i,+}(t)$ by assigning $x_{t}^{i}=X_{\varphi_{t}^{i}}^{i}$ and $x_{\bar{T}}^{i}=X_{\varphi_{\bar{T}}^{i}}^{i}$ to obtain the integral form of (B.15). Solution to (B.14) is found by substituting (A.5)-(A.6) in (B.15) and combining with the solution of (B.11). In turn, this provides the result for a call option on a forward zero-coupon bond under the SMMM as (7.2).

For a put option on a forward zero-coupon bond $P^{i}(\bar{T}, T)$ it is advantageous to separate the solution into two cases because of restrictions imposed by parameters (7.6)-(7.7). In the case of the bond binary put option on a forward zero-coupon bond one obtains

$$
\begin{aligned}
B_{\bar{T}, K, P^{i}(\bar{T}, T)}^{i,-}(t) & =E\left[\frac{S_{t}^{i, \delta_{*}}}{S_{\bar{T}}^{i, \delta_{*}}} \mathbb{1}_{\left\{P^{i}(\bar{T}, T)<K\right\}} \mid \mathcal{A}_{t}\right] \\
& = \begin{cases}P^{i}(t, \bar{T})-B_{\bar{T}, K, P^{i}(\bar{T}, T)}^{i,+}(t) & \text { for } \quad K<G_{T}^{i}(\bar{T}) \\
P^{i}(t, \bar{T}) & \text { for } \quad K \geq G_{T}^{i}(\bar{T})\end{cases}
\end{aligned}
$$

for $0 \leq t \leq \bar{T} \leq T$ and $i \in\{0,1, \ldots, d\}$. The solution to (B.16) when $K<$ $G_{T}^{i}(\bar{T})$ comes from the price of a zero-coupon bond (4.5) and (B.11), whilst when $K \geq G_{T}^{i}(\bar{T})$ the solution simplifies to (4.5). The asset binary put option on a forward zero-coupon bond is found as

$$
\begin{aligned}
A_{\bar{T}, K, P^{i}(\bar{T}, T)}^{i,-}(t) & =E\left[\frac{S_{t}^{i, \delta_{*}}}{S_{\bar{T}}^{i, \delta_{*}}} P^{i}(\bar{T}, T) \mathbb{1}_{\left\{P^{i}(\bar{T}, T) \leq K\right\}} \mid \mathcal{A}_{t}\right] \\
& = \begin{cases}P^{i}(t, T)-A_{\bar{T}, K, P^{i}(\bar{T}, T)}^{i,+}(t) & \text { for } \quad K<G_{T}^{i}(\bar{T}) \\
P^{i}(t, T) & \text { for } K \geq G_{T}^{i}(\bar{T})\end{cases}
\end{aligned}
$$

for $0 \leq t \leq \bar{T} \leq T$ and $i \in\{0,1, \ldots, d\}$. Thus combination of (B.16) and (B.17) lead to the expression for a put option a forward zero-coupon bond under the SMMM as shown in (7.3). 


\section{Acknowledgement}

The authors would like to thank Nicole El-Karoui, Hardy Hulley, David Heath, Erik Schlögl, and Leah Kelly for their interest in this research and stimulating and constructive discussions on the subject. The World Stock Accumulation Index (WSAI) data was obtained from Global Financial Data.

\section{References}

Black, F. (1976). Studies in stock price volatility changes. In Proceedings of the 1976 Business Meeting of the Business and Economic Statistics Section, pp. 177-181. American Statistical Association.

Black, F. \& M. Scholes (1973). The pricing of options and corporate liabilities. Journal of Political Economy 81, 637-654.

Brigo, D. \& F. Mercurio (2006). Interest Rate Models - Theory and Practice (Second ed.). Springer Finance.

Buchen, P. (2003). The pricing of dual expiry exotics. Quantitative Finance 3, $1-8$.

Delbaen, F. \& W. Schachermayer (1994). A general version of the fundamental theorem of asset pricing. Mathematische Annalen 300(3), 463-520.

Dimson, E., P. Marsh, \& M. Staunton (2002). Triumph of the Optimists: 101 Years of Global Investment Returns. Princeton University Press.

Ding, C. G. (1992). Algorithm AS275: Computing the non-central $\chi^{2}$ distribution function. Applied Statistics 41(2), 478-482.

Harrison, J. M. \& D. M. Kreps (1979). Martingale and arbitrage in multiperiod securities markets. Journal of Economic Theory 20(3), 381-408.

Heath, D., R. Jarrow, \& A. Morton (1992). Bond pricing and the term structure of interest rates: a new methodology. Econometrica 60(1), 77-105.

Heath, D. \& E. Platen (2002). Perfect hedging of index derivatives under a minimal market model. International Journal of Theoretical and Applied Finance 5(7), 757-774.

Heath, D. \& E. Platen (2005). Currency derivatives under a minimal market model with random scaling. International Journal of Theoretical and Applied Finance 8(8), 1157-1177.

Heath, D. \& E. Platen (2006). Local volatility function models under a benchmark approach. Quantitative Finance 6(3), 197-206.

Hulley, H., S. M. Miller, \& E. Platen (2005). Benchmarking and fair pricing applied to two market models. Kyoto Economic Review 74(1), 85-118. 
Johnson, N. L., S. Kotz, \& N. Balakrishnan (1995). Continuous Univariate Distributions (Second ed.), Volume 2. John Wiley \& Sons.

Jones, M. C. (1987). On the relationship between the Poisson-exponential model and the non-central chi-squared distribution. Scandinavian Actuarial Journal, 104-109.

Karatzas, I. \& S. E. Shreve (1998). Methods of Mathematical Finance. Springer Verlag.

Kelly, J. R. (1956). A new interpretation of the information rate. Bell Systems Technology Journal 35, 917-926.

Lewis, A. L. (2000). Option Valuation Under Stochastic Volatility (With Mathematica Code). Finance Press, Newport Beach, California.

Loewenstein, M. \& G. A. Willard (2000). Local martingales, arbitrage and viability: Free snacks and cheap thrills. Economic Theory 16, 135-161.

Long, J. B. (1990). The numeraire portfolio. Journal of Financial Economics 26, 29-69.

Miller, S. M. \& E. Platen (2005). A two-factor model for low interest rate regimes. Asia-Pacific Financial Markets 11(1), 107-133.

Platen, E. (1997). A non-linear stochastic volatility model. Working Paper, Australian National University, Financial Mathematics Research Reports, FMRR 005-97.

Platen, E. (2001). A minimal financial market model. In Trends in Mathematics, pp. 293-301. Birkhäuser.

Platen, E. (2002). Arbitrage in continuous complete markets. Advances in Applied Probability 34(3), 540-558.

Platen, E. (2005a). An alternative interest rate term structure model. International Journal of Theoretical and Applied Finance 8(6), 717-735.

Platen, E. (2005b). Diversified portfolios with jumps in a benchmark framework. Asia-Pacific Financial Markets 11(1), 1-22.

Platen, E. \& D. Heath (2006). A Benchmark Approach to Quantitative Finance. Springer Finance.

Revuz, D. \& M. Yor (1999). Continuous Martingales and Brownian Motion (Third ed.). Springer-Verlag.

Siegel, A. F. (1979). The noncentral chi-squared distribution with zero degrees of freedom and testing for uniformity. Biometrika 66(2), 381-386.

Torgerson, E. N. (1972). Supplementary notes on linear models. Statistical Memoirs I, University of Oslo, Institute of Mathematics.

Yor, M. (1992). On some exponential functionals of Brownian motion. Advances in Applied Probability 24, 509-531. 